\title{
Silylative Dieckmann-like Cyclizations of Ester-Imides (and Diesters)
}

\author{
Thomas R. Hoye*, Vadims Dvornikovs, Elena Sizova \\ Department of Chemistry, University of Minnesota, Minneapolis, MN 55455 \\ hoye@chem.umn.edu
}

\section{Supporting Information}

Spectroscopic characterization data and procedures for preparation of all new compounds $(\mathbf{4 , 6}$, $\mathbf{1 2 - 1 5}, \mathbf{1 7 - 2 1}, \mathbf{2 4}, \mathbf{2 5}, \mathbf{2 8}$, and 31; 25 pages). Intermediates that are new compounds and that were synthesized in the course of preparing the above numbered compounds are also described here. These have been given the Roman numerals I-VI.

\section{General Experimental Protocols.}

All glassware was flame- or/and oven-dried before use. Tetrahydrofuran (THF) and dichloromethane were dried through $\mathrm{Al}_{2} \mathrm{O}_{3}$ columns. $\mathrm{CH}_{3} \mathrm{CN}$ and EtOAc were distilled over $\mathrm{P}_{2} \mathrm{O}_{5}$ and stored over $3 \AA \mathrm{MS}$. $\mathrm{MeOH}$ was distilled over $\mathrm{Mg}$ and stored over $3 \AA \mathrm{MS}$. Et $\mathrm{E}_{3} \mathrm{~N}$ was distilled from $\mathrm{CaH}_{2}$ and stored over $4 \AA$ MS. Other solvents were of HPLC grade and were used as received. Analytical TLC was performed using TLC plastic sheets with $\mathrm{F}_{254}$ indicator, and detection was performed by UV-light or staining with either potassium permanganate or anisaldehyde $/ \mathrm{H}_{2} \mathrm{SO}_{4}$.

Most purifications were performed on a medium pressure chromatography unit using columns packed with 25-32 $\mu \mathrm{m}$ silica gel. Each unit is outfitted with a Waters HPLC pump and refractive index detector.

${ }^{1} \mathrm{H}$ and ${ }^{13} \mathrm{C}$ NMR spectra were acquired on a Varian VI-500 $\left(500 \mathrm{MHz}{ }^{1} \mathrm{H}, 125.7 \mathrm{MHz}{ }^{13} \mathrm{C}\right)$, VI$300\left(300 \mathrm{MHz}{ }^{1} \mathrm{H}, 75.4 \mathrm{MHz}{ }^{13} \mathrm{C}\right)$, or VXR-300 (300 MHz $\left.{ }^{1} \mathrm{H}, 75.4 \mathrm{MHz}{ }^{13} \mathrm{C}\right)$ spectrometer, using $\mathrm{CDCl}_{3}$ or $\mathrm{C}_{6} \mathrm{D}_{6}$. Chemical shifts $(\delta)$ for spectra recorded in $\mathrm{CDCl}_{3}$ are reported in ppm and are referenced to TMS at $\delta=0.00$ for ${ }^{1} \mathrm{H}$ and $\mathrm{CDCl}_{3}$ at $\delta=77.23 \mathrm{ppm}$ for ${ }^{13} \mathrm{C}$ NMR spectra. The following abbreviations are used to describe multiplicities: s (singlet), $\mathrm{d}$ (doublet), $\mathrm{t}$ (triplet), $\mathrm{q}$ (quartet), pent (pentet), m (multiplet), nform (non-first order multiplet), app (apparent), and br (broad). Coupling constants $(J)$ are reported in Hz.

Infrared spectra were recorded on a Midac Corporation Prospect 4000 FT-IR spectrometer (only the most intense and/or diagnostic peaks are reported). All samples were thin films on a $\mathrm{NaCl}$ window.

High-resolution mass spectra were recorded on a Bruker Biotof II (ESI-TOF) instrument using PEG or PPG as an internal calibrant. Low resolution GC-MS data were collected on an Agilent 5975 XL-MSD instrument at $70 \mathrm{eV}$ fitted with an HP-5 column ( $\sim 15 \mathrm{~m}$ in length). 


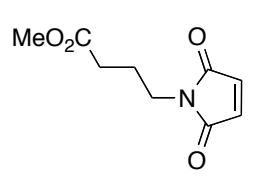

2

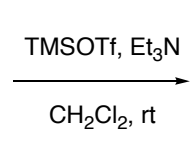

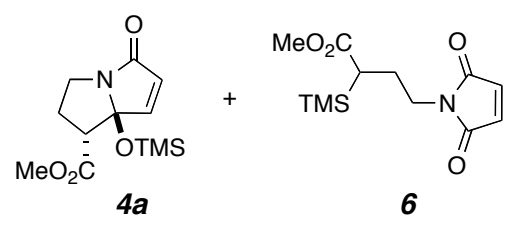

Methyl (1R,7aS)-rel-2,3,4,7a-Tetrahydro-7a-[(trimethylsilyl)oxy]-5(1H)-pyrrolizinone-1carboxylate (4a) and Methyl 2,5-Dihydro-2,5-dioxo-1 $H$-pyrrole-1- $(\alpha-$ trimethylsilyl)butanoate (6).

Imide ester 2 (376 mg, $1.9 \mathrm{mmol})$ was dissolved in $\mathrm{CH}_{2} \mathrm{Cl}_{2}(7.0 \mathrm{~mL}) . \mathrm{Et}_{3} \mathrm{~N}(0.66 \mathrm{~mL}, 4.7 \mathrm{mmol})$ and TMSOTf $(0.69 \mathrm{~mL}, 3.8 \mathrm{mmol})$ were added sequentially at rt. The reaction mixture was stirred for $4 \mathrm{~h}$, diluted with $\mathrm{CH}_{2} \mathrm{Cl}_{2}(20 \mathrm{~mL})$, and washed sequentially with a saturated aqueous solution of $\mathrm{NaHCO}_{3}$ and brine. The organic layer was dried over $\mathrm{Na}_{2} \mathrm{SO}_{4}$. The solution was concentrated and the residue was purified by silica gel chromatography with hexanes/EtOAc 3:1 as eluent. This provided, in order of elution, $6(21 \mathrm{mg}, 0.08 \mathrm{mmol}, 4 \%)$ as white crystals and $4 \mathbf{a}$ (dr 62:1 (GC-MS), $450 \mathrm{mg}, 1.67 \mathrm{mmol}, 88 \%$ ) as white crystals.

For 6: $\mathrm{mp}=70-71{ }^{\circ} \mathrm{C}$.

${ }^{1} \mathrm{H}-\mathrm{NMR}\left(300 \mathrm{MHz}, \mathrm{CDCl}_{3}\right.$ ): $\delta 6.70$ (s, 2H), 3.65 (s, 3H), 3.55 (app t, 2H, $J=7 \mathrm{~Hz}$ ), 2.24 (dddd, $1 \mathrm{H}, J=13.8,12.1,6.2,6.2 \mathrm{~Hz}), 1.94(\mathrm{dd}, 1 \mathrm{H}, J=12.1,2.4 \mathrm{~Hz}), 1.70$ (dddd, $1 \mathrm{H}, J=13.7,7.1$, 7.1, $2.3 \mathrm{~Hz})$, and $0.05(\mathrm{~s}, 9 \mathrm{H})$.

${ }^{13} \mathrm{C}-\mathrm{NMR}\left(75 \mathrm{MHz}, \mathrm{CDCl}_{3}\right): \delta 175.0,171.0,134.3,51.4,38.1,34.8,25.6$, and 4.1.

TLC: $\mathrm{R}_{\mathrm{f}}($ hexanes/EtOAc, $3: 1)=0.33$.

IR: 2955, 1712, 1407, 1251, and $1192 \mathrm{~cm}^{-1}$.

MS: ESI-MS calcd for $\mathrm{C}_{12} \mathrm{H}_{19} \mathrm{NNaO}_{4} \mathrm{Si}\left(\mathrm{M} \cdot \mathrm{Na}^{+}\right)$292.0976, found 292.0973.

GC-MS (Start temp $=50{ }^{\circ} \mathrm{C}$, run time $=21 \mathrm{~min}$, temp. $\left.\operatorname{ramp}=11.4{ }^{\circ} \mathrm{C} / \mathrm{min}\right): \mathrm{t}_{\mathrm{R}}=10.3 \mathrm{~min} ; \mathrm{m} / \mathrm{z}$ : $269\left(\mathrm{M}^{+}\right), 254\left(\mathrm{M}^{+}-\mathrm{CH}_{3}\right)$.

A sample for single crystal $\mathrm{x}$-ray analysis was obtained by preparing a saturated solution of 6 in hexanes followed by slow cooling to $-20{ }^{\circ} \mathrm{C}$. The solution was kept for $20 \mathrm{~h}$ at $-20{ }^{\circ} \mathrm{C}$. Needlelike crystals were collected by filtration.

For 4a: $\mathrm{mp}=64.5-66^{\circ} \mathrm{C}$

${ }^{1} \mathrm{H}-\mathrm{NMR}\left(300 \mathrm{MHz}, \mathrm{CDCl}_{3}\right): \delta 6.97(\mathrm{~d}, 1 \mathrm{H}, J=5.7 \mathrm{~Hz}), 5.95(\mathrm{dd}, 1 \mathrm{H}, J=5.7,1.0 \mathrm{~Hz}), 3.76$ (ddd, $1 \mathrm{H}, J=11.8,8.9,8.9 \mathrm{~Hz}), 3.56(\mathrm{~s}, 3 \mathrm{H}), 3.27$ (ddd, $1 \mathrm{H}, J=11.3,9.1,2.4 \mathrm{~Hz}), 3.11$ (dd, 1H, $J=6.9,1.2 \mathrm{~Hz}$ ), 2.60 (dddd, $1 \mathrm{H}, J=13.2,9.2,9.2,6.8 \mathrm{~Hz}), 2.56(\mathrm{dddd}, 1 \mathrm{H}, J=12.9,8.3,2,2$ $\mathrm{Hz})$, and $0.09(\mathrm{~s}, 9 \mathrm{H})$.

${ }^{13} \mathrm{C}-\mathrm{NMR}\left(75 \mathrm{MHz}, \mathrm{CDCl}_{3}\right): \delta 173.9,171.5,148.3,127.8,101.0,53.0,52.1,42.3,31.3$, and 1.3. TLC: $\mathrm{R}_{\mathrm{f}}($ hexanes/EtOAc, $3: 1)=0.30$.

IR: $3067,2957,2905,1732,1713,1485,1433,1356$, and $1321 \mathrm{~cm}^{-1}$.

MS: ESI-MS calcd for $\mathrm{C}_{12} \mathrm{H}_{19} \mathrm{NNaO}_{4} \mathrm{Si}\left(\mathrm{M} \cdot \mathrm{Na}^{+}\right)$292.0976, found 292.0965.

GC-MS (Start temp $=50{ }^{\circ} \mathrm{C}$, run time $=21 \mathrm{~min}$, temp. $\left.\operatorname{ramp}=11.4{ }^{\circ} \mathrm{C} / \mathrm{min}\right): \mathrm{t}_{\mathrm{R}}=10.1 \mathrm{~min} ; \mathrm{m} / \mathrm{z}$ : $269\left(\mathrm{M}^{+}\right), 254\left(\mathrm{M}^{+}-\mathrm{CH}_{3}\right)$. 


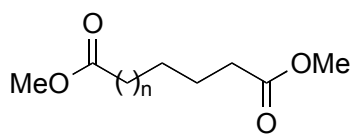

$11 a n=1$

$11 b \mathrm{~b}=2$
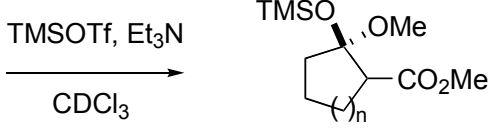

$12 a \mathrm{n}=1$

$12 b n=2$

\section{Methyl cis- and trans-2-Methoxy-2-trimethylsilyloxycyclopentanecarboxylates (12a).}

Dimethyl adipate $11 \mathrm{a}(31.2 \mathrm{mg}, 0.18 \mathrm{mmol})$ was dissolved in $\mathrm{CDCl}_{3}(0.5 \mathrm{~mL})$ and $\mathrm{Et}_{3} \mathrm{~N}(65 \mu \mathrm{L}$, $0.45 \mathrm{mmol})$ was added followed by TMSOTf $(63 \mu \mathrm{L}, 0.36 \mathrm{mmol})$. The resulting mixture was shaken to achieve adequate mixing and after $3 \mathrm{~h}$ filtered through a bed of silica gel with 80:20:1 hexanes/EtOAc/ $\mathrm{Et}_{3} \mathrm{~N}$ to give an orange oil (46 mg, 86\%, dr 2:1). This labile diastereomeric mixture of 12a was $\sim 95 \%$ pure $\left({ }^{1} \mathrm{H}\right.$ NMR analysis $)$.

TLC: $\mathrm{R}_{\mathrm{f}}\left(\right.$ hexanes/EtOAc, $\left.4: 1+1 \% \mathrm{Et}_{3} \mathrm{~N}\right)=0.55$.

${ }^{1} \mathrm{H}-\mathrm{NMR}\left(500 \mathrm{MHz}, \mathrm{CDCl}_{3}\right.$ ): $\delta 3.68$ (s, 3H, minor), 3.67 (s, 3H, major), 3.28 (s, 3H, major), 3.27 (s, 3H, minor), 3.03 (dd, 1H, $J=8.3,5.5 \mathrm{~Hz}$, major), 2.94 (dd, 1H, $J=8.5,4.6 \mathrm{~Hz}$, minor), 2.02 (dddd, $1 \mathrm{H}, J=13,8,7,4 \mathrm{~Hz}$ (from 1D TOCSY), major), 1.80 (dddd, $1 \mathrm{H}, J=12,12,8,4 \mathrm{~Hz}$ (from 1D-TOCSY), major), 1.58-2.06 (m), 0.19 (s, 9H, minor), 0.13 (s, 9H, major).

${ }^{13} \mathrm{C}-\mathrm{NMR}\left(75 \mathrm{MHz}, \mathrm{CDCl}_{3}\right): \delta 173.9,173.8,110.8,110.3,54.2,51.5,51.3,51.1,50.8,49.1$, $38.5,36.2,26.67,26.61,22.5,21.3,1.4$, and 1.2.

IR: 2956, 1740, 1436, 1351, 1252, 844, and $757 \mathrm{~cm}^{-1}$.

MS: ESI-MS calcd for $\mathrm{C}_{11} \mathrm{H}_{22} \mathrm{O}_{4} \mathrm{SiNa}\left(\mathrm{M} \bullet \mathrm{Na}^{+}\right)$269.1180, found 269.1185.

\section{Methyl cis- and trans-2-Methoxy-2-trimethylsilyloxycyclohexanecarboxylates (12b).}

Esters 12b (an ca. 2:1 mixture of diastereomers, yellow oil, 98\%) was obtained using the procedure above (for 12a) with the exception that the reaction mixture was allowed to stand for $24 \mathrm{~h}$ before workup.

TLC: $\mathrm{R}_{\mathrm{f}}\left(\right.$ Hexanes/EtOAc, $\left.4: 1+1 \% \mathrm{Et}_{3} \mathrm{~N}\right)=0.39$

${ }^{1} \mathrm{H}-\mathrm{NMR}\left(500 \mathrm{MHz}, \mathrm{CDCl}_{3}\right.$ ): $\delta 3.67$ (s, 3H, minor), 3.65 (s, 3H, major), 3.220 (s, 3H, major), 3.217 (s, 3H, minor), 2.89 (ddd, $1 \mathrm{H}, J=4.4,4.4,1.8 \mathrm{~Hz}$, major), 2.73 (ddd, $1 \mathrm{H}, J=4.9,4.9,1.1$ $\mathrm{Hz}$, minor), 2.14 (m, 1H, minor), 2.11 (ddd, 1H, $J=13.3,11.5,4.2 \mathrm{~Hz},(1 \mathrm{D}-\mathrm{TOCSY}$ ) major), 1.86-1.32 (m), $0.18(\mathrm{~s}, 9 \mathrm{H}$, minor), and $0.12(\mathrm{~s}, 9 \mathrm{H}$, major).

${ }^{13} \mathrm{C}-\mathrm{NMR}\left(75 \mathrm{MHz}, \mathrm{CDCl}_{3}\right.$ ): $\delta$ 173.2, 172.9, 99.6, 51.2, 51.1, 48.4, 48.1, 48.0, 33.6, 26.4, 26.2, $22.8,22.1,21.0,1.74$, and 1.71 .

IR: 2951, 2865, 1741, 1436, 1366, 1341, 1251, 1158, 1105, 1057, 1021, and $843 \mathrm{~cm}^{-1}$.

MS: ESI-MS calcd for $\mathrm{C}_{11} \mathrm{H}_{22} \mathrm{O}_{4} \mathrm{SiNa}\left(\mathrm{M} \bullet \mathrm{Na}^{+}\right)$283.1336, found 283.1346. 

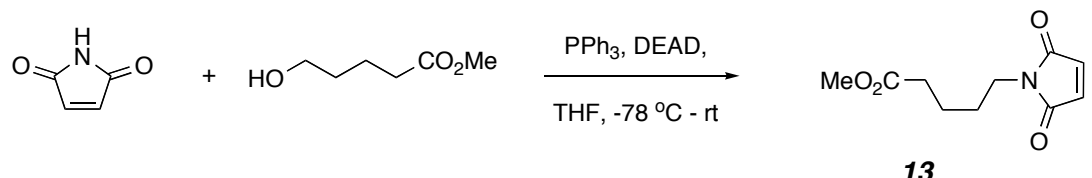

Methyl 2,5-Dihydro-2,5-dioxo-1H-pyrrole-1-pentanoate (13).

A solution of $\mathrm{Ph}_{3} \mathrm{P}(0.393 \mathrm{~g}, 1.50 \mathrm{mmol})$ in THF $(11 \mathrm{~mL})$ was cooled to $-78^{\circ} \mathrm{C}$. Diethyl diazodicarboxylate $(1.10 \mathrm{~mL}, 1.50 \mathrm{mmol})$ was added over $2 \mathrm{~min}$. The yellow reaction mixture was stirred for $5 \mathrm{~min}$. Methyl 5-hydroxypentanoate $10.200 \mathrm{~g}, 1.50 \mathrm{mmol})$ was added over $5 \mathrm{~min}$ and the reaction mixture was stirred for $5 \mathrm{~min}$. Maleimide $(0.147 \mathrm{~g}, 1.50 \mathrm{mmol})$ was added to the reaction mixture as a solid. The resulting solution was allowed to remain at $-78{ }^{\circ} \mathrm{C}$ for $5 \mathrm{~min}$, the cooling bath was removed, and the solution was stirred at ambient temperature for $48 \mathrm{~h}$ and concentrated in vacuo. Purification of the resulting residue by chromatography on silica gel with hexanes/EtOAc 3:1 as eluent gave pure $13(0.153 \mathrm{~g}, 0.73 \mathrm{mmol}, 48 \%)$ as a colorless oil:

TLC: $\mathrm{R}_{\mathrm{f}}($ hexanes/EtOAc, $3: 1)=0.21$

${ }^{1} \mathrm{H}-\mathrm{NMR}\left(500 \mathrm{MHz}, \mathrm{CDCl}_{3}\right): \delta 6.70(\mathrm{~s}, 2 \mathrm{H}), 3.67$ (s, 3H), 3.54 (t, 2H, J=6.5 Hz), 2.35 (t, 2H, $J$ $=7.0 \mathrm{~Hz}), 1.59-1.67$ (4H, nfom).

${ }^{13} \mathrm{C}-\mathrm{NMR}\left(125 \mathrm{MHz}, \mathrm{CDCl}_{3}\right): \delta 173.8,171.0,134.3,51.8,37.6,33.6,28.1$, and 22.2.

IR: $3100,2952,2871,1737,1706,1441,1410,1368,1197,1175,830$, and $696 \mathrm{~cm}^{-1}$.

MS: ESI-MS calcd for $\mathrm{C}_{10} \mathrm{H}_{13} \mathrm{NO}_{4} \mathrm{~K}\left(\mathrm{M} \bullet \mathrm{K}^{+}\right)$250.0476, found 250.0474; for $\mathrm{C}_{10} \mathrm{H}_{13} \mathrm{NO}_{4} \mathrm{Na}$ $\left(\mathrm{M} \bullet \mathrm{Na}^{+}\right)$234.0737, found 234.0749. 


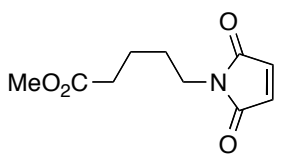

13

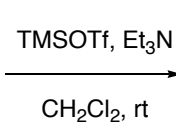

$\mathrm{CH}_{2} \mathrm{Cl}_{2}$, rt

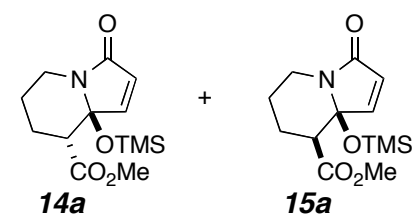

$15 a$

Methyl (1R,8aS)-rel- and (1R,8aR)-rel-2,3,4,8a-Tetrahydro-8a-[(trimethylsilyl)oxy]-6(5H)indolizinone-1-carboxylate (14a and 15a).

Imide ester 13 (137 mg, $0.65 \mathrm{mmol})$ was dissolved in $\mathrm{CH}_{2} \mathrm{Cl}_{2}(3 \mathrm{~mL})$. $\mathrm{Et}_{3} \mathrm{~N}(361 \mu \mathrm{L}, 2.60 \mathrm{mmol})$ and TMSOTf $(353 \mu \mathrm{L}, 1.95 \mathrm{mmol})$ were added sequentially to the reaction mixture at $0{ }^{\circ} \mathrm{C}$. The reaction mixture was warmed to $\mathrm{rt}$ and stirred for $14 \mathrm{~h}$. $\mathrm{Et}_{3} \mathrm{~N}(136 \mu \mathrm{L}, 0.98 \mathrm{mmol})$ and TMSOTf $(120 \mu \mathrm{L}, 0.65 \mathrm{mmol})$ were added. The resulting mixture was stirred at $\mathrm{rt}$ for $4 \mathrm{~h}$, diluted with $\mathrm{CH}_{2} \mathrm{Cl}_{2}(50 \mathrm{~mL})$, and washed sequentially with a saturated aqueous solution of $\mathrm{NaHCO}_{3}$ and brine. Organic layer was dried over $\mathrm{Na}_{2} \mathrm{SO}_{4}$. Concentration under reduced pressure yielded a 5.5:1 mixture of 14a:15a (GC, ${ }^{1} \mathrm{H}$ NMR), which was chromatographed on silica gel with $\mathrm{CH}_{2} \mathrm{Cl}_{2} / \mathrm{MeOH}$ (50:1 eluent).

Pure 14a (118 mg, $0.42 \mathrm{mmol}, 50 \%$, slower eluting) was obtained as a colorless oil:

TLC: $\mathrm{R}_{\mathrm{f}}$ (hexanes/EtOAc, 3:1) $=0.20$.

${ }^{1} \mathrm{H}-\mathrm{NMR}\left(300 \mathrm{MHz}, \mathrm{CDCl}_{3}\right): \delta 6.91(\mathrm{~d}, 1 \mathrm{H}, J=5.7 \mathrm{~Hz}), 6.16(\mathrm{~d}, 1 \mathrm{H}, J=6.0 \mathrm{~Hz}), 4.12(\mathrm{dd}, 1 \mathrm{H}, J$ $=13.6,4.9 \mathrm{~Hz}), 3.55(\mathrm{~s}, 3 \mathrm{H}), 3.03(\mathrm{dd}, 1 \mathrm{H}, J=4.8,2.4 \mathrm{~Hz}), 2.89(\mathrm{ddd}, 1 \mathrm{H}, J=12.7,12.7,3.9$ $\mathrm{Hz}$ ), 2.15 (dddd, $1 \mathrm{H}, J=12.9,12.9,4.5,4.5 \mathrm{~Hz}$ ), 1.95 (ddddd, $1 \mathrm{H}, J=13,13,13,5.4,3.3 \mathrm{~Hz}$ ), 1.94-1.84 (br d, $1 \mathrm{H}, J=13 \mathrm{~Hz}$ ), 1.57-1.47 (nfom, 1H), and 0.07 (s, 9H).

${ }^{13} \mathrm{C}-\mathrm{NMR}\left(75 \mathrm{MHz}, \mathrm{CDCl}_{3}\right): \delta 171.4,167.1,147.6,128.4,88.7,51.7,49.0,35.5,21.9,19.9$, and 1.6.

IR: $2956,1740,1714,1409,1253,1075$, and $860 \mathrm{~cm}^{-1}$.

MS: ESI-MS calcd for $\mathrm{C}_{13} \mathrm{H}_{22} \mathrm{NO}_{4} \mathrm{Si}\left(\mathrm{M} \bullet \mathrm{H}^{+}\right)$284.1313, found 284.1314.

Pure 15a (22 mg, $0.06 \mathrm{mmol}, 9 \%$, faster eluting) was obtained as a colorless oil:

TLC: $\mathrm{R}_{\mathrm{f}}($ hexanes/EtOAc, $3: 1)=0.20$.

${ }^{1} \mathrm{H}-\mathrm{NMR}\left(300 \mathrm{MHz}, \mathrm{CDCl}_{3}\right): \delta 7.51(\mathrm{~d}, 1 \mathrm{H}, J=5.7 \mathrm{~Hz}), 6.19(\mathrm{~d}, 1 \mathrm{H}, J=6.0 \mathrm{~Hz}), 4.10$ (ddd, $1 \mathrm{H}, J=13.2,5.1,1.5,1.5 \mathrm{~Hz}), 3.75$ (s, 3H), 2.81 (ddd, 1H, $J=12.9,12.9,3.6 \mathrm{~Hz}), 2.25$ (dd, 1H, $J=12.3,4.5 \mathrm{~Hz}$ ), 2.07 (dddd, $1 \mathrm{H}, J=13,13,13,3.6 \mathrm{~Hz}$ ), 1.96 (ddddd, 1H, $J=13.5,4,4,4,1.5$ $\mathrm{Hz}$ ), 1.79 (ddddd, $1 \mathrm{H}, J=13.5,3.3,3.3,3.3,1.5 \mathrm{~Hz}$ ), 1.31 (ddddd, $1 \mathrm{H}, J=13.3,13.3,13.3,5.1$, $4.4 \mathrm{~Hz})$, and $0.04(\mathrm{~s}, 9 \mathrm{H})$.

${ }^{13} \mathrm{C}-\mathrm{NMR}\left(75 \mathrm{MHz}, \mathrm{CDCl}_{3}\right): \delta 171.1,150.4,127.1,89.4,55.4,55.1,29.9,24.9,22.0$, and 1.39. IR: 2956, 2926, 1744, 1710, 1407, 1253, 1126, and $846 \mathrm{~cm}^{-1}$.

MS: ESI-MS calcd for $\mathrm{C}_{13} \mathrm{H}_{21} \mathrm{NNaO}_{4} \mathrm{Si}\left(\mathrm{M} \bullet \mathrm{Na}^{+}\right)$306.1132, found 306.1134. 


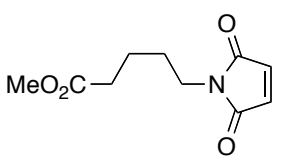

13

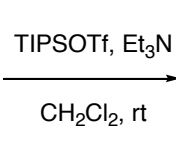

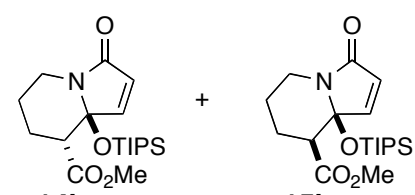

$15 b$

Methyl (1R,8aS)-rel- and $(1 R, 8 \mathrm{a} R)-r e l-2,3,4,8 \mathrm{a}-$ Tetrahydro-8a-[[tris(1methylethyl)silyl]oxy]-6(5H)-indolizinone-1-carboxylate (14b and 15b).

Imide ester 13 (38 mg, $0.18 \mathrm{mmol})$ was dissolved in $\mathrm{CDCl}_{3}(0.6 \mathrm{~mL})$. $\mathrm{Et}_{3} \mathrm{~N}$ (55 $\left.\mu \mathrm{L}, 0.40 \mathrm{mmol}\right)$ and TIPSOTf $(97 \mu \mathrm{L}, 0.36 \mathrm{mmol})$ were added sequentially to the reaction mixture at $\mathrm{rt}$. The reaction mixture was stirred for $4 \mathrm{~h}$, and additional $\mathrm{Et}_{3} \mathrm{~N}(25 \mu \mathrm{L}, 0.18 \mathrm{mmol})$ and TIPSOTf (45 $\mu \mathrm{L}, 0.18 \mathrm{mmol}$ ) were added. The resulting mixture was stirred at $\mathrm{rt}$ for $15 \mathrm{~h}$, diluted with $\mathrm{CH}_{2} \mathrm{Cl}_{2}$ $(20 \mathrm{~mL})$, and washed sequentially with saturated aqueous solution of $\mathrm{NaHCO}_{3}$ and brine. Organic layer was dried over $\mathrm{Na}_{2} \mathrm{SO}_{4}$. The solution was concentrated and purified by silica gel chromatography with hexanes/EtOAc 3:1 as eluent. Both diastereomers 14b:15b (dr 7.5:1, 52 $\mathrm{mg}, 0.14 \mathrm{mmol}, 78 \%$ ) coeluted and were obtained as a colorless oil.

For 14b:

${ }^{1} \mathrm{H}-\mathrm{NMR}\left(500 \mathrm{MHz}, \mathrm{CDCl}_{3}\right): \delta 6.92(\mathrm{~d}, 1 \mathrm{H}, J=6.0 \mathrm{~Hz}), 6.19(\mathrm{~d}, 1 \mathrm{H}, J=6.5 \mathrm{~Hz}), 4.13$ (br dd, $1 \mathrm{H}, J=12.5,5.5 \mathrm{~Hz}), 3.56(\mathrm{~s}, 3 \mathrm{H}), 3.10(\mathrm{dd}, 1 \mathrm{H}, J=4.5,2.0 \mathrm{~Hz}), 3.02(\mathrm{ddd}, 1 \mathrm{H}, J=13.0,13.0$, $4.0 \mathrm{~Hz}$ ), 2.23 (dddd, 1H, $J=13.0,13.0,4.0,4.0 \mathrm{~Hz}$ ), 1.99 (ddddd, $1 \mathrm{H}, J=13.0,13.0,13.0,5.0$, $4.0 \mathrm{~Hz}$ ), 1.92 (ddddd, $1 \mathrm{H}, J=13.0,4.0,2.5,2.5,1.5 \mathrm{~Hz}$ ), 1.53 (ddddd, $1 \mathrm{H}, J=13.0,4.0,4.0,2.5$, $1.5 \mathrm{~Hz}), 1.05(\mathrm{~m}, 9 \mathrm{H})$, and $1.01(\mathrm{~m}, 9 \mathrm{H})$.

${ }^{13} \mathrm{C}-\mathrm{NMR}\left(125 \mathrm{MHz}, \mathrm{CDCl}_{3}\right): \delta 171.2,166.6,146.4,129.0,88.0,51.6,49.4,35.4,21.8,19.7$, 18.1, 18.0, and 13.0.

For the mixture: TLC: $\mathrm{R}_{\mathrm{f}}$ (hexanes/EtOAc, 3:1) $=0.20$.

IR: $2947,2868,1741,1715,1408,1149,1083$, and $1064 \mathrm{~cm}^{-1}$.

MS: ESI-MS calcd for $\mathrm{C}_{19} \mathrm{H}_{34} \mathrm{NO}_{4} \mathrm{Si}\left(\mathrm{M} \cdot \mathrm{H}^{+}\right)$368.2252, found 368.2249. 

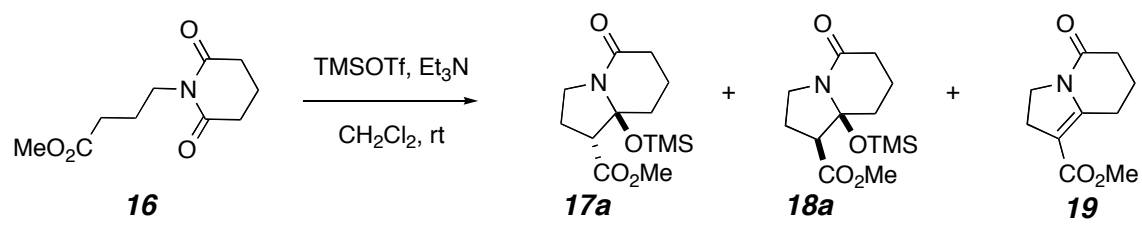

Methyl $(1 R, 8 \mathrm{a} S)$-rel-1,2,3,5,6,7,8,8a-Octahydro-8a-[trismethylsilyl]oxy-5-oxo-(1H)indolizinecarboxylate (17a), Methyl $(1 R, 8 a R)$-rel-1,2,3,5,6,7,8,8a-Octahydro-8a[trismethylsilyl]oxy-5-oxo-(1H)-indolizinecarboxylate (18a), and Methyl 2,3,5,6,7,8Hexahydro-5-oxo-1-indolizinecarboxylate (19).

Imide ester $\mathbf{1 6}^{\mathbf{2}}(160 \mathrm{mg}, 0.75 \mathrm{mmol})$ was dissolved in $\mathrm{CH}_{2} \mathrm{Cl}_{2}(2 \mathrm{~mL})$. $\mathrm{Et}_{3} \mathrm{~N}(173 \mu \mathrm{L}, 1.24$ mmol) and TMSOTf $(180 \mu \mathrm{L}, 1.00 \mathrm{mmol})$ were added sequentially at $0{ }^{\circ} \mathrm{C}$. The reaction mixture was warmed to $\mathrm{rt}$, and after $10 \mathrm{~h} \mathrm{Et}_{3} \mathrm{~N}(90 \mu \mathrm{L}, 0.65 \mathrm{mmol})$ and $\operatorname{TMSOTf}(90 \mu \mathrm{L}, 0.50 \mathrm{mmol})$ were added again. After $4 \mathrm{~h}$ the resulting mixture was diluted with $\mathrm{CH}_{2} \mathrm{Cl}_{2}(50 \mathrm{~mL})$ and washed sequentially with saturated aqueous solution of $\mathrm{NaHCO}_{3}$ and brine. Organic layer was dried over $\mathrm{Na}_{2} \mathrm{SO}_{4}$. Concentration under reduced pressure yielded a mixture of 17a, 18a, and 19 in 2:1:6 ratio, respectively. Chromatography on silica gel with hexanes/EtOAc 1:1 as eluent gave three pure components, listed in the order of elution: 19 (29 $\mathrm{mg}, 0.15 \mathrm{mmol}, 20 \%)$ as white crystals, 17a (13 mg, $0.08 \mathrm{mmol}, 11 \%)$ as a colorless oil, 18a (139 $\mathrm{mg}, 0.49 \mathrm{mmol}, 65 \%)$ as a colorless oil.

For 19:

$\mathrm{mp}=91-94{ }^{\circ} \mathrm{C}$.

TLC: $\mathrm{R}_{\mathrm{f}}($ hexanes/EtOAc, $1: 1)=0.20$.

${ }^{1} \mathrm{H}-\mathrm{NMR}\left(500 \mathrm{MHz}, \mathrm{CDCl}_{3}\right): \delta 3.88(\mathrm{t}, 2 \mathrm{H}, J=9.5 \mathrm{~Hz}), 3.74(\mathrm{~s}, 3 \mathrm{H}), 2.97(\mathrm{t}, 2 \mathrm{H}, J=6.5 \mathrm{~Hz})$, $2.77(\mathrm{t}, 2 \mathrm{H}, J=9.5 \mathrm{~Hz}), 2.49(\mathrm{t}, 2 \mathrm{H}, J=6.5 \mathrm{~Hz})$, and $1.88(\mathrm{tt}, 2 \mathrm{H}, J=6.5,6.5 \mathrm{~Hz})$.

${ }^{13} \mathrm{C}-\mathrm{NMR}\left(125 \mathrm{MHz}, \mathrm{CDCl}_{3}\right): \delta 168.9,166.5,152.5,108.4,51.3,44.5,32.4,26.7,23.6$, and 19.5 .

IR: 2954, 1694, 1675, 1621, 1407,1260, 1241, and $1186 \mathrm{~cm}^{-1}$.

MS: ESI-MS calcd for $\mathrm{C}_{10} \mathrm{H}_{14} \mathrm{NO}_{3}\left(\mathrm{M} \bullet \mathrm{H}^{+}\right)$196.0968, found 196.0960.

For 17a:

TLC: $\mathrm{R}_{\mathrm{f}}($ hexanes/EtOAc, $1: 1)=0.15$.

${ }^{1} \mathrm{H}-\mathrm{NMR}\left(500 \mathrm{MHz}, \mathrm{CDCl}_{3}\right.$ ): $\delta 3.84$ (ddd, $\left.1 \mathrm{H}, J=12.0,8.5,8.5 \mathrm{~Hz}\right), 3.68(\mathrm{~s}, 3 \mathrm{H}), 3.54$ (ddd, 1H, $J=12.0,10.0,3.0 \mathrm{~Hz}), 3.18(\mathrm{dd}, 1 \mathrm{H}, J=7.5,1.0 \mathrm{~Hz}), 2.45(\mathrm{dddd}, 1 \mathrm{H}, J=18.0,6.5,1.5,1.5 \mathrm{~Hz}$ ), 2.26 (ddd, $1 \mathrm{H}, J=18.5,12.0,7.5 \mathrm{~Hz}$ ), 2.20 (dddd, 1H, $J=13.0,9.5,9.0,7.5 \mathrm{~Hz}$ ), 2.00-2.14 (nfom, 3H), 1.78 (ddddd, $1 \mathrm{H}, J=13.0,7.5,3.5,3.5,2.0 \mathrm{~Hz}$ ), 1.49 (ddd, 1H, $J=13.5,13.5,4.0$ $\mathrm{Hz})$, and $0.16(\mathrm{~s}, 9 \mathrm{H})$.

Pure 18a TLC: $\mathrm{R}_{\mathrm{f}}($ hexanes/EtOAc, $1: 1)=0.11$

${ }^{1} \mathrm{H}-\mathrm{NMR}\left(500 \mathrm{MHz}, \mathrm{CDCl}_{3}\right): \delta 3.74$ (s, 3H, OMe), 3.59-3.55 (m, $2 \mathrm{H}, \mathrm{H} 3 \beta$ and $\mathrm{H} 3 \alpha$ ), $2.84(\mathrm{dd}, 1 \mathrm{H}, J=12.0,8.0 \mathrm{~Hz}, \mathrm{H} 1 \alpha), 2.52-2.39$ (m, 3H, H2, H6 $\beta, \mathrm{H} 8 \beta$ ), 2.32 (ddd, $1 \mathrm{H}, J=18.5,11.5,7.5, \mathrm{H} 6 \alpha$ ), 2.06 (dddd, $1 \mathrm{H}, J=13.5,8,7,3.5 \mathrm{~Hz}, \mathrm{H} 2$ ), 1.99 (dddd, $1 \mathrm{H}, J=$

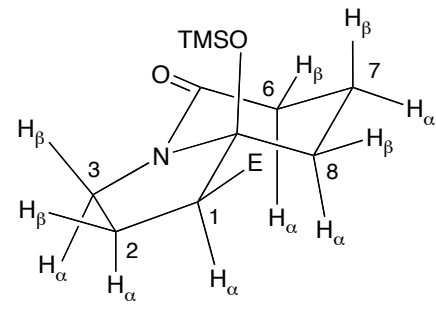

18a $\left(\mathrm{E}=\mathrm{CO}_{2} \mathrm{Me}\right)$ 
15.0, 14.0, 11, 7.0, $3.5 \mathrm{~Hz}, \mathrm{H} 7 \beta$ ), 1.85 (ddddd, $1 \mathrm{H}, J=13,7.5,3.5,3.5,1.0 \mathrm{~Hz}, \mathrm{H} 7 \alpha$ ), 1.60 (ddd, $1 \mathrm{H}, J=13.5,13.5,4.0 \mathrm{~Hz}, \mathrm{H} 8 \alpha$ ), and 0.09 (s, 9H, TMS).

${ }^{1} \mathrm{H}-\mathrm{NMR}\left(500 \mathrm{MHz}, \mathrm{C}_{6} \mathrm{D}_{6}\right.$, referenced to residual $\mathrm{C}_{6} \mathrm{D}_{5} \mathrm{H}$ at $\delta$ 7.16): $\delta 3.45-3.34(\mathrm{~m}, 2 \mathrm{H}, \mathrm{H} 3 \beta$ and $\mathrm{H} 3 \alpha$ ), 3.29 (s, 3H, OMe), 2.33-2.21 (m, 4H, H2, H6 $\beta, \mathrm{H} 8 \beta, \mathrm{H} 1 \alpha$ ), 2.00 (ddd, $1 \mathrm{H}, J=19.0$, 12.0, 7.5, H6 $\alpha$ ), 1.69 (dddd, $1 \mathrm{H}, J=15.0,14.0,11.5,6.5,3.5 \mathrm{~Hz}, \mathrm{H} 7 \beta$ ), 1.48 (nfom, $1 \mathrm{H}, \mathrm{H} 2$ ), 1.23 (ddddd, $1 \mathrm{H}, J=14.0,7.5,3.5,3.5,1.5 \mathrm{~Hz}, \mathrm{H} 7 \alpha$ ), 1.06 (ddd, $1 \mathrm{H}, J=13.5,13.5,3.5 \mathrm{~Hz}$, $\mathrm{H} 8 \alpha$ ), and 0.02 (s, 9H, TMS).

${ }^{13} \mathrm{C}-\mathrm{NMR}\left(75 \mathrm{MHz}, \mathrm{CDCl}_{3}\right): \delta 170.1,169.9,91.3,56.6,52.0,44.0,35.3,31.1,21.9,16.9$, and 1.5 .

IR: 2954, 1741, 1655, 1400,1253, 1042, and $842 \mathrm{~cm}^{-1}$.

MS: ESI-MS calcd for $\mathrm{C}_{13} \mathrm{H}_{24} \mathrm{NO}_{4} \mathrm{Si}\left(\mathrm{M} \bullet \mathrm{H}^{+}\right)$286.1469, found 286.1471 . 

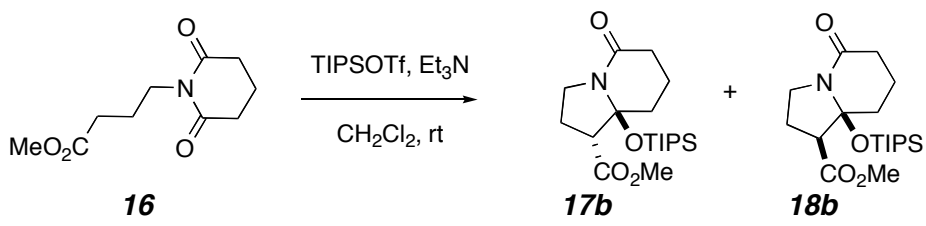

Methyl (1R, 8aS)-rel-1,2,3,5,6,7,8,8a-octahydro-8a-[tris(1-methylethyl)silyl]oxy-5-oxo-(1H)indolizinecarboxylate (17b) and methyl $(1 R, 8 \mathrm{a} R)$-rel-1,2,3,5,6,7,8,8a-octahydro-8a-[tris(1methylethyl)silyl]oxy-5-oxo-(1H)-indolizinecarboxylate (18b).

Imide ester 16 (160 mg, $0.75 \mathrm{mmol})$ was dissolved in $\mathrm{CH}_{2} \mathrm{Cl}_{2}(2 \mathrm{~mL}) . \mathrm{Et}_{3} \mathrm{~N}(173 \mu \mathrm{L}, 1.24 \mathrm{mmol})$ and TIPSOTf $(267 \mu \mathrm{L}, 1.00 \mathrm{mmol})$ were added sequentially to the reaction mixture at $0{ }^{\circ} \mathrm{C}$. The reaction mixture was warmed to rt, stirred for $10 \mathrm{~h}$, then additional $\mathrm{Et}_{3} \mathrm{~N}(90 \mu \mathrm{L}, 0.65 \mathrm{mmol})$ and TMSOTf $(135 \mu \mathrm{L}, 0.50 \mathrm{mmol})$ were added. The resulting mixture was stirred at $\mathrm{rt}$ for $7 \mathrm{~h}$, diluted with $\mathrm{CH}_{2} \mathrm{Cl}_{2}(50 \mathrm{~mL})$, and washed sequentially with saturated aqueous solution of $\mathrm{NaHCO}_{3}$ and brine. Organic layer was dried over $\mathrm{Na}_{2} \mathrm{SO}_{4}$. Concentration under reduced pressure yielded a mixture of $\mathbf{1 7 b}$ and $\mathbf{1 8 b}$ in 1.1:1 ratio, respectively. Chromatography on silica gel with hexanes/EtOAc 1:1 as eluent gave two pure components, listed in the order of elution: 17b (128 $\mathrm{mg}, 0.35 \mathrm{mmol}, 50 \%)$ as colorless oil, $\mathbf{1 8 b}(119 \mathrm{mg}, 0.32 \mathrm{mmol}, 45 \%)$ as a colorless oil.

Pure 17b TLC: $\mathrm{R}_{\mathrm{f}}$ (hexanes/EtOAc, 1:1) $=0.25$.

${ }^{1} \mathrm{H}-\mathrm{NMR}\left(300 \mathrm{MHz}, \mathrm{CDCl}_{3}\right.$ ): $\delta 3.94$ (ddd, $\left.1 \mathrm{H}, J=11.7,8.7,8.7 \mathrm{~Hz}\right), 3.65$ (s, 3H), 3.50 (ddd, 1H, $J=12.6,9.6,3.6 \mathrm{~Hz}), 3.18(\mathrm{dd}, 1 \mathrm{H}, J=7.6,1.9 \mathrm{~Hz}), 2.42(\mathrm{ddd}, 1 \mathrm{H}, J=17.4,7.0,2.4 \mathrm{~Hz}), 2.29-$

1.97 (nfom, $5 \mathrm{H}$ ), 1.70 (ddddd, $1 \mathrm{H}, J=11.0,7.2,4.2,4.2,2.8 \mathrm{~Hz}), 1.57$ (ddd, $1 \mathrm{H}, J=12.8,12.8$, $3.7 \mathrm{~Hz}$ ), and 1.04 (br s, 21H).

${ }^{13} \mathrm{C}-\mathrm{NMR}\left(75 \mathrm{MHz}, \mathrm{CDCl}_{3}\right): \delta$ 172.7, 169.5, 92.3, 57.0, 52.1, 44.1, 32.9, 31.0, 23.9, 18.3, and 13.8 .

IR: $2949,2868,1738,1661,1403,1210$, and $1099 \mathrm{~cm}^{-1}$.

MS: ESI-MS calcd for $\mathrm{C}_{19} \mathrm{H}_{36} \mathrm{NO}_{4} \mathrm{Si}\left(\mathrm{M} \bullet \mathrm{H}^{+}\right)$370.2408, found 370.2416.

Pure 18b TLC: $\mathrm{R}_{\mathrm{f}}$ (hexanes/EtOAc, 1:1) $=0.20$.

${ }^{1} \mathrm{H}-\mathrm{NMR}\left(500 \mathrm{MHz}, \mathrm{CDCl}_{3}\right): \delta 3.80(\mathrm{ddd}, 1 \mathrm{H}, J=12.0,9.0,9.0 \mathrm{~Hz}$, H3), 3.72 (s, 3H, OMe), $3.48(\mathrm{ddd}, 1 \mathrm{H}, J=12.5,10.0,3.0 \mathrm{~Hz}, \mathrm{H} 3)$, 2.77 (dd, $1 \mathrm{H}, J=12.0,8.5 \mathrm{~Hz}, \mathrm{H} 1 \alpha), 2.55$ (dddd, $1 \mathrm{H}, J=13.0,11.5$, 10.0, $9.0 \mathrm{~Hz}, \mathrm{H} 2$ ), 2.49-2.43 (m, 2H, H6, H8), 2.33 (ddd, 1H, $J=$ 18.0, 9.5, 6.0 Hz, H6), 2.12-2.04 (m, 2H, H8, H2), 2.01(m, 1H, H7), 1.78 (nfom, 1H, H7), and 1.05-1.01 (m, 21H).

${ }^{13} \mathrm{C}-\mathrm{NMR}\left(75 \mathrm{MHz}, \mathrm{CDCl}_{3}\right): \delta 170.4,170.3,92.3,56.9,52.4,43.6$,

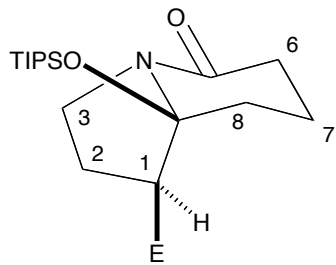
$18 b\left(\mathrm{E}=\mathrm{CO}_{2} \mathrm{Me}\right)$ $35.2,32.5,22.8,18.8,18.7,18.3$, and 14.4.

IR: $2948,2868,1741,1654,1403,1182$, and $1045 \mathrm{~cm}^{-1}$.

MS: ESI-MS calcd for $\mathrm{C}_{19} \mathrm{H}_{36} \mathrm{NO}_{4} \mathrm{Si}\left(\mathrm{M} \bullet \mathrm{H}^{+}\right)$370.2408, found 370.2415. 


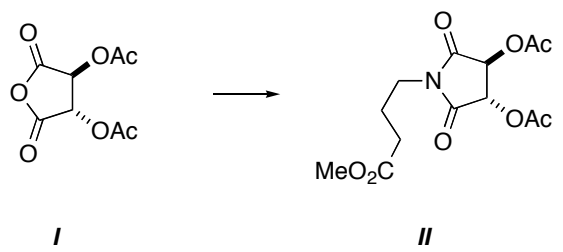

\section{Methyl (3S,4S)-3,4-bis(Acetyloxy)-2,5-dioxo-1-pyrrolidinebutanoate (II).}

Known D-anhydride $\mathbf{I}^{3}\left(1.47 \mathrm{~g}, 6.8 \mathrm{mmol}\right.$; prepared from D-tartaric acid and $\left.\mathrm{AcCl}^{3}\right)$ was dissolved in $\mathrm{CH}_{3} \mathrm{CN}$. The hydrochloride salt of methyl 4-aminobutyrate ${ }^{4}(1.10 \mathrm{~g}, 7.2 \mathrm{mmol})$ was added followed by $\mathrm{Et}_{3} \mathrm{~N}(1.0 \mu \mathrm{L}, 7.2 \mathrm{mmol})$. The reaction mixture was stirred for $2 \mathrm{~h}$ at room temperature, and the solvent was removed in vacuo. Acetyl chloride $(10 \mathrm{~mL})$ was added and the reaction mixture was stirred for $2 \mathrm{~h}$ at room temperature. Excess acetyl chloride was removed by distillation (at one atmosphere) and the residue was chromatographed on silica gel with hexanes/EtOAc 2:1 as eluent. Pure II (1.48 g, $4.7 \mathrm{mmol}, 69 \%)$ was obtained as a pale yellow oil having an $[\alpha]_{\mathrm{D}}=-68.6^{\circ}\left(\mathrm{c} 8.15, \mathrm{CHCl}_{3}\right)$. We also prepared a sample of the L-antipode of II starting from L-tartaric acid. The rotation of this sample of L-II was: $[\alpha]_{D}=+66.5^{\circ}(\mathrm{c} 0.53$, $\left.\mathrm{CHCl}_{3}\right)$. The L-antipode is reported ${ }^{5}$ to have a rotation of: $[\alpha]_{\mathrm{D}}=-57.2^{\circ}\left(\mathrm{c} 1.0, \mathrm{CHCl}_{3}\right)$. A search of the Beilstein database revealed other $N$-alkylated tartarimide bisesters whose specific rotation values are reported; all examples are consistent in having a positive value for each (of 6) of the L-compounds and a negative value for each (of 2) of the D-compounds.

TLC: $\mathrm{R}_{\mathrm{f}}$ (hexanes/EtOAc, $\left.1: 1\right)=0.50$.

${ }^{1} \mathrm{H}-\mathrm{NMR}\left(500 \mathrm{MHz}, \mathrm{CDCl}_{3}\right): \delta 5.50(\mathrm{~s}, 2 \mathrm{H}), 3.72-3.62(\mathrm{~m}, 2 \mathrm{H}), 3.68(\mathrm{~s}, 3 \mathrm{H}), 2.38(\mathrm{t}, 2 \mathrm{H}, 7.2$ $\mathrm{Hz}), 2.20(\mathrm{~s}, 6 \mathrm{H})$, and 2.02-1.93 (m, 2H).

${ }^{13} \mathrm{C}-\mathrm{NMR}\left(75 \mathrm{MHz}, \mathrm{CDCl}_{3}\right): \delta 173.2,170.1,169.6,72.9,51.9,38.9,31.2,22.7$, and 20.5. IR: 2958, ( 1750 sh), 1728, 1440, 1406, 1374, 1221, and $1060 \mathrm{~cm}^{-1}$.

MS: ESI-MS calcd for $\mathrm{C}_{13} \mathrm{H}_{17} \mathrm{NO}_{8} \mathrm{Na}\left(\mathrm{M} \bullet \mathrm{Na}^{+}\right)$338.0846, found 338.0842. 


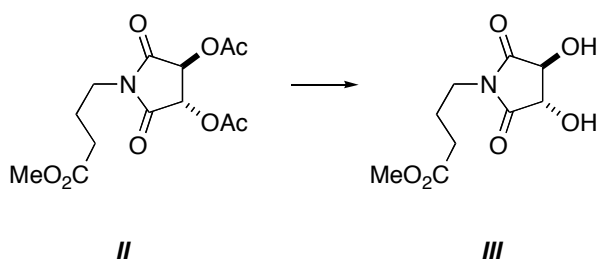

Methyl (3S,4S)-3,4-Dihydroxy-2,5-dioxo-1-pyrrolidinebutanoate (III).

bis-Acetoxy D-tartarimide II (163 mg, $0.52 \mathrm{mmol})$ was dissolved in methanol (15 $\mathrm{mL})$ and acetyl chloride $(10 \mu \mathrm{L})$ was added via a syringe while stirring. The resulting reaction mixture was refluxed for $24 \mathrm{~h}$ and concentrated under reduced pressure to produce a white solid (104 $\mathrm{mg}$, $0.45 \mathrm{mmol}, 87 \%$ ), which was essentially pure III by ${ }^{1} \mathrm{H}$ NMR and TLC analyses. This material had an $[\alpha]_{D}=-111.4^{\circ}(\mathrm{c} 1.04, \mathrm{MeOH})$. We also prepared a sample of the L-antipode of III starting from L-tartaric acid (by way of the L-antipode of II). The rotation of this sample of L III was: $[\alpha]_{\mathrm{D}}=+107.7^{\circ}[\mathrm{c} 0.49, \mathrm{MeOH}]$. The L-antipode (i.e., L-III) has been previously prepared and was reported ${ }^{5}$ to have a rotation of: $[\alpha]_{D}=-101.6^{\circ}(\mathrm{c} 1.0, \mathrm{MeOH})$. A search of the Beilstein database revealed other $N$-alkylated tartarimide diols whose specific rotation values are reported; all examples are consistent in having a positive value for each (of 6) of the Lcompounds and a negative value for each (of 2) of the D-compounds.

TLC: $\mathrm{R}_{\mathrm{f}}($ EtOAc $)=0.57$.

$\mathrm{mp}=86-90{ }^{\circ} \mathrm{C}$.

${ }^{1} \mathrm{H}-\mathrm{NMR}\left(500 \mathrm{MHz}, \mathrm{CDCl}_{3}\right): \delta 4.61$ (s, 2H), 3.68 (s, 3H), 3.65 (dt, 1H, 13.2, $\left.6.7 \mathrm{~Hz}\right), 3.58$ (dt, $1 \mathrm{H}, 13.6,6.9 \mathrm{~Hz}$ ), 3.26 (br s, $2 \mathrm{H}$, variable in chemical shift from sample to sample), 2.37 (t, $2 \mathrm{H}$, $7.1 \mathrm{~Hz}$ ), 1.97 (ddddd, 1H, 13.7, 7, 7, 7, $7 \mathrm{~Hz}$ ), and 1.94 (ddddd, 1H, 14.1, 7, 7, 7, $7 \mathrm{~Hz}$ ).

${ }^{13} \mathrm{C}-\mathrm{NMR}\left(75 \mathrm{MHz}, \mathrm{CDCl}_{3}\right.$ ): $\delta$ 175.0, 173.9, 74.9, 52.2, 38.4, 31.3, and 22.8.

IR: 3434 (br), 2954, and $1709 \mathrm{~cm}^{-1}$.

MS: ESI-MS calcd for $\mathrm{C}_{9} \mathrm{H}_{13} \mathrm{NO}_{6} \mathrm{Na}\left(\mathrm{M} \bullet \mathrm{Na}^{+}\right)$254.0635, found 254.0627. 


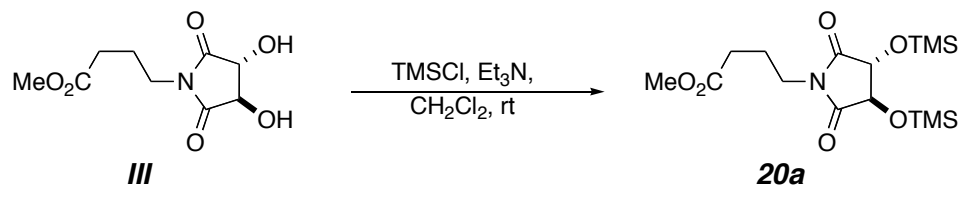

\section{Methyl (3R,4R)-2,5-Dioxo-3,4-bis[(trimethylsilyl)oxy]-1-pyrrolidinebutanoate (20a).}

To a stirred solution of L-diol III $(0.300 \mathrm{~g}, 1.30 \mathrm{mmol})$ in $\mathrm{CH}_{2} \mathrm{Cl}_{2}(15 \mathrm{~mL})$ under a $\mathrm{N}_{2}$ atmosphere $\mathrm{Et}_{3} \mathrm{~N}(0.55 \mathrm{~mL}, 3.90 \mathrm{mmol})$ and $\mathrm{TMSCl}(0.46 \mathrm{~mL}, 3.6 \mathrm{mmol})$ were added sequentially at $0^{\circ} \mathrm{C}$. The resulting solution was warmed up to rt, stirred for $14 \mathrm{~h}$, diluted with $\mathrm{CH}_{2} \mathrm{Cl}_{2}(30 \mathrm{~mL})$, and washed with a saturated solution of $\mathrm{NaHCO}_{3}(10 \mathrm{~mL})$. Organic layer was separated and aqueous layer was extracted back with $\mathrm{CH}_{2} \mathrm{Cl}_{2}$. Organic layers were combined, washed with brine, dried over $\mathrm{Na}_{2} \mathrm{SO}_{4}$ and concentrated. The obtained residue was purified by silica gel chromatography with hexanes/EtOAc 6:1 as eluent to give the L-imide ester 20a $(0.380$ $\mathrm{g}, 78 \%)$ as a colorless oil having an $[\alpha]_{\mathrm{D}}=+113.9^{\circ}\left(\mathrm{c} 0.79, \mathrm{CHCl}_{3}\right)$. We also prepared the Dantipode ent-20a (structure not shown). Its rotation was: $[\alpha]_{\mathrm{D}}=-109.6^{\circ}\left(\mathrm{c} 1.70, \mathrm{CHCl}_{3}\right)$. The Lantipode (i.e., 20a) is reported ${ }^{5}$ to have a rotation of: $[\alpha]_{\mathrm{D}}=-109.6^{\circ}$ (c 1.0, $\mathrm{CHCl}_{3}$ ). A search of the Beilstein database revealed two other $N$-alkylated tartarimide bis-silyl ethers whose specific rotation values are reported; both of these -compounds have a positive value.

TLC: $\mathrm{R}_{\mathrm{f}}$ (hexanes/EtOAc, 6:1) $=0.35$.

${ }^{1} \mathrm{H}-\mathrm{NMR}\left(500 \mathrm{MHz}, \mathrm{CDCl}_{3}\right.$ ): $\delta 4.43$ (s, 2H), 3.67 (s, 3H), 3.59 (ddd, 1H, $J=13.3,6.7,6.7 \mathrm{~Hz}$ ), 3.51 (ddd, $1 \mathrm{H}, J=13.4,7.0,7.0 \mathrm{~Hz}$ ), 2.33 (t, 2H, $J=7.3 \mathrm{~Hz}$ ), 2.10 (ddddd, $1 \mathrm{H}, J=14.4,7.2$, 7.2, 7.2, 7.2 Hz), 1.90 (ddddd, $1 \mathrm{H}, J=13.9,7.5,7.5,7.5,7.5 \mathrm{~Hz}$ ), and $0.24(\mathrm{~s}, 18 \mathrm{H})$.

${ }^{13} \mathrm{C}-\mathrm{NMR}\left(125 \mathrm{MHz}, \mathrm{CDCl}_{3}\right): \delta 173.5,173.2,76.9,52.0,38.2,31.6,23.1$, and 0.2.

IR: 2957, 2902, 1727, 1362, 12531164,1133 , and $847 \mathrm{~cm}^{-1}$.

MS: ESI-MS calcd for $\mathrm{C}_{15} \mathrm{H}_{29} \mathrm{NNaO}_{6} \mathrm{Si}_{2}\left(\mathrm{M} \cdot \mathrm{Na}^{+}\right) 398.1426$, found 398.1426. 


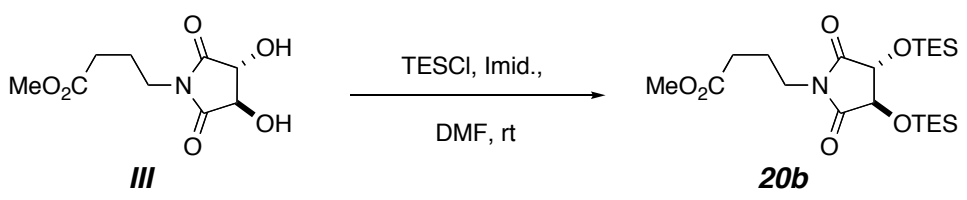

\section{Methyl (3R,4R)-2,5-Dioxo-3,4-bis[(triethylsilyl)oxy]-1-pyrrolidinebutanoate (20b).}

To a stirred solution of L-diol III $(0.150 \mathrm{~g}, 0.650 \mathrm{mmol})$ in DMF $(2 \mathrm{~mL})$ under a $\mathrm{N}_{2}$ atmosphere imidazole $(0.133 \mathrm{~g}, 1.96 \mathrm{mmol})$ and $\mathrm{TESCl}(0.255 \mathrm{~g}, 1.69 \mathrm{mmol})$ were added sequentially. The resulting solution was stirred for $48 \mathrm{~h}$ at ambient temperature and poured into ice-cold water (10 $\mathrm{mL})$, which was then extracted with EtOAc $(3 \times 10 \mathrm{~mL})$. The combined organic layers were washed with brine $(2 \times 10 \mathrm{~mL})$, dried over $\mathrm{Na}_{2} \mathrm{SO}_{4}$, and concentrated in vacuo. The obtained residue was purified by silica gel chromatography with hexanes/EtOAc 6:1 as eluent to give the L-imide ester 20b (0.178 g, 59\%) as a colorless oil.

$[\alpha]_{\mathrm{D}}=+109.8^{\circ}\left(\mathrm{c} 0.52, \mathrm{CHCl}_{3}\right)$.

TLC: $\mathrm{R}_{\mathrm{f}}($ hexanes/EtOAc, $6: 1)=0.32$.

${ }^{1} \mathrm{H}-\mathrm{NMR}\left(500 \mathrm{MHz}, \mathrm{CDCl}_{3}\right.$ ): $\delta 4.46$ (s, 2H), 3.67 (s, 3H), 3.58 (ddd, 1H, $J=13.7,7.0,7.0 \mathrm{~Hz}$ ), 3.50 (ddd, $1 \mathrm{H}, J=13.6,6.9,6.9 \mathrm{~Hz}$ ), 2.34 (t, 2H, $J=7.2 \mathrm{~Hz}$ ), 2.10 (ddddd, 1H, $J=14.4,7.1,7.1$, 7.1, 7.1 Hz), 2.05 (ddddd, $1 \mathrm{H}, J=14.1,7.1,7.1,7.1,7.1 \mathrm{~Hz}), 1.00$ (t, 18H, $J=7.8 \mathrm{~Hz}$ ), 0.90 (dq, $6 \mathrm{H}, J=15.4,7.5 \mathrm{~Hz})$, and $0.86(\mathrm{dq}, 6 \mathrm{H}, J=15.7,8.0 \mathrm{~Hz})$.

${ }^{13} \mathrm{C}-\mathrm{NMR}\left(125 \mathrm{MHz}, \mathrm{CDCl}_{3}\right): \delta 173.7,173.2,77.0,51.9,38.1,31.6,23.1,6.8$, and 5.0.

IR: 2956, 2914, 2879, 1725, 1164, 1133, and $743 \mathrm{~cm}^{-1}$.

MS: ESI-MS calcd for $\mathrm{C}_{21} \mathrm{H}_{41} \mathrm{NNaO}_{6} \mathrm{Si}_{2}\left(\mathrm{M} \cdot \mathrm{Na}^{+}\right)$482.2365, found 482.2357. 

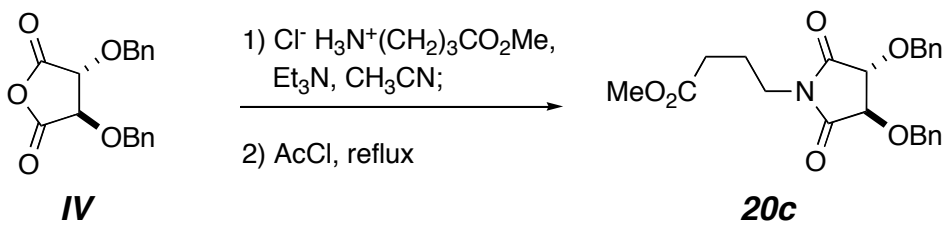

\section{Methyl (3R,4R)-2,5-dioxo-3,4-bis(benzyloxy)-1-pyrrolidinebutanoate (20b).}

To a stirred suspension of methyl $\gamma$-aminobutyrate hydrochloride $(0.307 \mathrm{~g}, 2.00 \mathrm{mmol})$ and Lanhydride $\mathbf{I V}^{6}$ in $\mathrm{CH}_{3} \mathrm{CN}(20 \mathrm{~mL}), \mathrm{Et}_{3} \mathrm{~N}(0.278 \mathrm{~mL}, 2.00 \mathrm{mmol})$ was added. This solution was stirred for $3 \mathrm{~h}$ at $\mathrm{rt}$ and concentrated under reduced pressure. To the resulting residue $\mathrm{AcCl}$ (20 $\mathrm{mL}$ ) was added, and the solution was heated under reflux overnight, cooled to rt, and filtered through a $2 \mathrm{~cm}$ layer of silica gel with EtOAc. The filtrate was concentrated and purified by silica gel chromatography with hexanes/EtOAc 3:1 as eluent, yielding the L-imide 20c $(0.501 \mathrm{~g}$, $61 \%)$ as a colorless oil.

$$
[\alpha]_{\mathrm{D}}=+98.3^{\circ}\left(\mathrm{c} 0.23, \mathrm{CDCl}_{3}\right) \text {. }
$$

TLC: $\mathrm{R}_{\mathrm{f}}$ (hexanes/EtOAc, 3:1) $=0.30$.

${ }^{1} \mathrm{H}-\mathrm{NMR}\left(500 \mathrm{MHz}, \mathrm{CDCl}_{3}\right): \delta 7.37-7.31(\mathrm{~m}, 10 \mathrm{H}), 4.98(\mathrm{~d}, 2 \mathrm{H}, J=11.0 \mathrm{~Hz}), 4.75(\mathrm{~d}, 2 \mathrm{H}, J=$ $11.5 \mathrm{~Hz}), 4.38(\mathrm{~s}, 2 \mathrm{H}), 3.64(\mathrm{~s}, 3 \mathrm{H}), 3.60(\mathrm{ddd}, 1 \mathrm{H}, J=13.1,6.8,6.8 \mathrm{~Hz}), 3.53(\mathrm{ddd}, 1 \mathrm{H}, J=$ 13.7, 7.2, $7.2 \mathrm{~Hz}$ ), 2.33 (t, 2H, $J=7.1 \mathrm{~Hz}$ ), 1.94 (ddddd, 1H, $J=14.0,7.1,7.1,7.1,7.1 \mathrm{~Hz}$ ), 1.88 (ddddd, 1H, $J=13.9,7.1,7.1,7.1,7.1 \mathrm{~Hz}$ ).

${ }^{13} \mathrm{C}-\mathrm{NMR}\left(125 \mathrm{MHz}, \mathrm{CDCl}_{3}\right): \delta 172.9,172.8,136.6,128.6,128.3,78.8,73.5,51.8,38.0,31.3$, and 22.8 .

IR: 3064, 3032, 2951, 2876, 1724, 1349, 1121, and $1079 \mathrm{~cm}^{-1}$.

MS: ESI-MS calcd for $\mathrm{C}_{23} \mathrm{H}_{25} \mathrm{NNaO}_{6}\left(\mathrm{M} \cdot \mathrm{Na}^{+}\right)$434.1574, found 434.1584. 


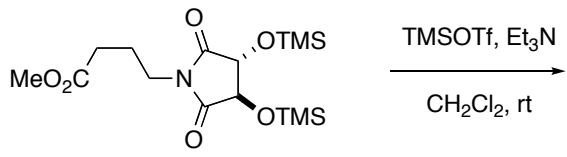

$20 a$

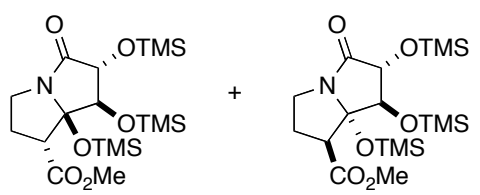

$21 a$

$22 a$

Methyl (1R,6R,7R,7aR)-7a-[(Trimethylsilyl)oxy]hexahydro-5-oxo-6,7bis[(trimethylsilyl)oxy]-1H-pyrrolizine-1-carboxylate (21a) and Methyl $(1 S, 6 R, 7 R, 7 \mathrm{a} S)-7 \mathrm{a}-$ [(Trimethylsilyl)oxy] hexahydro-5-oxo-6,7-bis[(trimethylsilyl)oxy]-1H-pyrrolizine-1carboxylate (22a).

L-Imide ester 20a (460 mg, $1.23 \mathrm{mmol})$ was dissolved in $\mathrm{CH}_{2} \mathrm{Cl}_{2}(5 \mathrm{~mL})$. $\mathrm{Et}_{3} \mathrm{~N}(683 \mu \mathrm{L}, 4.92$ $\mathrm{mmol})$ and TMSOTf $(666 \mu \mathrm{L}, 3.69 \mathrm{mmol})$ were added sequentially to the reaction mixture at 0 ${ }^{\circ} \mathrm{C}$. The mixture was warmed to $\mathrm{rt}$ and stirred for $16 \mathrm{~h}$. Additional $\mathrm{Et}_{3} \mathrm{~N}(256 \mu \mathrm{L}, 1.85 \mathrm{mmol})$ and TMSOTf $(222 \mu \mathrm{L}, 1.23 \mathrm{mmol})$ were added. The resulting mixture was stirred at $\mathrm{rt}$ for $6 \mathrm{~h}$ and diluted with $\mathrm{CH}_{2} \mathrm{Cl}_{2}(40 \mathrm{~mL})$. This solution was washed sequentially with saturated aqueous $\mathrm{NaHCO}_{3}$ and brine and dried over $\mathrm{Na}_{2} \mathrm{SO}_{4}$. GC-MS analysis of this crude mixture indicated a 23:1 ratio of two diastereomers. Chromatography on silica gel with hexanes/EtOAc 3:1 as eluent gave, in order of elution, 21a (383 mg, $0.86 \mathrm{mmol}, 70 \%)$ as colorless oil and 22a (19 mg, 0.05 mmol, $4 \%$ ) as white crystals.

21a TLC: $\mathrm{R}_{\mathrm{f}}$ (hexanes/EtOAc, 6:1) $=0.40$.

$[\alpha]_{\mathrm{D}}=+42.8^{\circ}\left(\mathrm{c} 0.9, \mathrm{CHCl}_{3}\right)$.

${ }^{1} \mathrm{H}-\mathrm{NMR}\left(300 \mathrm{MHz}, \mathrm{CDCl}_{3}\right.$ ): $\delta 4.44$ (ddd, $\left.1 \mathrm{H}, J=7.7,1.0,1.0 \mathrm{~Hz}\right), 3.89$ (d, $1 \mathrm{H}, J=7.6 \mathrm{~Hz}$ ), 3.74 (ddd, $1 \mathrm{H}, J=10.4,8.3,8.3 \mathrm{~Hz}), 3.68(\mathrm{~s}, 3 \mathrm{H}), 3.25(\mathrm{br} \mathrm{dd}, 1 \mathrm{H}, J=10.2,10.2 \mathrm{~Hz}), 3.02$ (dd, $1 \mathrm{H}, J=7.2,1.8 \mathrm{~Hz}$ ), 2.40 (dddd, $1 \mathrm{H}, J=13.5,9.5,9.5,7.4 \mathrm{~Hz}$ ), 2.24 (dddd, $1 \mathrm{H}, J=13.2,8.1$, 2.2, $2.2 \mathrm{~Hz}), 0.20$ (s, 9H), $0.17^{+}$(s, 9H), and $0.17^{-}(\mathrm{s}, 9 \mathrm{H})$.

${ }^{13} \mathrm{C}-\mathrm{NMR}\left(75 \mathrm{MHz}, \mathrm{CDCl}_{3}\right)$ : $\delta$ 172.1, 170.2, 95.9, 79.0, 77.6, 53.8, 52.2, 41.0, 28.6, 2.1, 0.7, and 0.5 .

IR: 2959, 2902, 1736, 1253, 1167, and $846 \mathrm{~cm}^{-1}$.

MS: ESI-MS calcd for $\mathrm{C}_{18} \mathrm{H}_{37} \mathrm{NNaO}_{6} \mathrm{Si}_{3}\left(\mathrm{M} \bullet \mathrm{Na}^{+}\right)$470.1821, found 470.1823 .

22a TLC: $\mathrm{R}_{\mathrm{f}}($ hexanes/EtOAc, $6: 1)=0.30$.

$[\alpha]_{\mathrm{D}}=-50.6^{\circ}\left(\mathrm{c} 0.17, \mathrm{CHCl}_{3}\right)$.

$\mathrm{mp}=70-73{ }^{\circ} \mathrm{C}$. TLC: $\mathrm{R}_{\mathrm{f}}($ hexanes/EtOAc, $6: 1)=0.30$

${ }^{1} \mathrm{H}-\mathrm{NMR}\left(500 \mathrm{MHz}, \mathrm{CDCl}_{3}\right.$ ): $\delta 4.09$ (d, $1 \mathrm{H}, J=6.2 \mathrm{~Hz}$ ), 3.87 (d, $1 \mathrm{H}, J=6.2 \mathrm{~Hz}$ ), 3.68 (ddd, $1 \mathrm{H}$, $J=11.4,8.6,8.6 \mathrm{~Hz}$ ), 3.67 (s, $3 \mathrm{H}), 3.29$ (ddd, $1 \mathrm{H}, J=11.4,9.4,1.5 \mathrm{~Hz}), 3.00$ (d, $1 \mathrm{H}, J=6.8$

$\mathrm{Hz}$ ), 2.54 (dddd, $1 \mathrm{H}, J=13.2,9.6,9.6,7.2 \mathrm{~Hz}$ ), 2.27 (dddd, $1 \mathrm{H}, J=13.1,7.9,1.9,1.2 \mathrm{~Hz}$ ), 0.18 (s, 9H), 0.17 (s, 9H), and $0.16(\mathrm{~s}, 9 \mathrm{H})$.

${ }^{13} \mathrm{C}-\mathrm{NMR}\left(75 \mathrm{MHz}, \mathrm{CDCl}_{3}\right): \delta 172.9,169.1,99.3,76.0,69.5,53.8,52.2,40.6,29.8,2.3,0.6$, and 0.4 .

IR: $2957,1724,1715,1250,1132$, and $840 \mathrm{~cm}^{-1}$.

MS: ESI-MS calcd for $\mathrm{C}_{18} \mathrm{H}_{37} \mathrm{NNaO}_{6} \mathrm{Si}_{3}\left(\mathrm{M} \bullet \mathrm{Na}^{+}\right)$470.1821, found 470.1836 . 


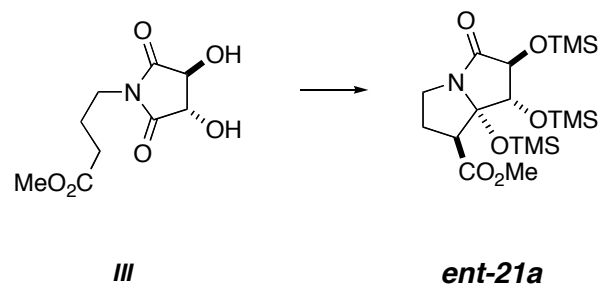

Methyl (1S,6S,7S,7aS)-7a-[(Trimethylsilyl)oxy]hexahydro-5-oxo-6,7bis[(trimethylsilyl)oxy]-1H-pyrrolizine-1-carboxylate (ent-21a).

D-Tartarimide III (142 mg, $0.61 \mathrm{mmol})$ was dissolved in $\mathrm{CH}_{2} \mathrm{Cl}_{2}(3 \mathrm{~mL}) . \mathrm{Et}_{3} \mathrm{~N}(430 \mu \mathrm{L}, 3.1$ mmol) was added at $\mathrm{rt}$ followed by TMSOTf $(440 \mu \mathrm{L}, 2.44 \mathrm{mmol})$. The reaction mixture was stirred for $24 \mathrm{~h}$ and partitioned between $\mathrm{CH}_{2} \mathrm{Cl}_{2}(50 \mathrm{~mL})$ and $5 \%$ aqueous solution of $\mathrm{NaHCO}_{3}$. The aqueous layer was extracted with $\mathrm{CH}_{2} \mathrm{Cl}_{2}(3 \times 25 \mathrm{~mL})$ and the combined organic layers were dried over $\mathrm{Na}_{2} \mathrm{SO}_{4}$. Concentration of the extract under reduced pressure yielded a brown residue that was chromatographed on silica gel with hexanes/EtOAc 6:1 as eluent to provide ent-21a (204 mg, $0.46 \mathrm{mmol}, 75 \%$ ) was obtained as a pale yellow oil.

$[\alpha]_{\mathrm{D}}=-42.4^{\circ}\left(\mathrm{c} 1.36, \mathrm{CHCl}_{3}\right)$.

TLC: $\mathrm{R}_{\mathrm{f}}($ hexanes/EtOAc, $4: 1)=0.50$.

${ }^{1} \mathrm{H}-\mathrm{NMR}\left(500 \mathrm{MHz}, \mathrm{CDCl}_{3}\right): \delta 4.44$ (br d, 1H, $\left.7.6 \mathrm{~Hz}\right), 3.90$ (d, 1H, $\left.7.5 \mathrm{~Hz}\right), 3.76$ (ddd, 1H, 10.6, 8.5, 8.5 Hz), 3.69 (s, 3H), 3.27 (dddd, 1H, 10.8, 9.1, 2.3, $1.3 \mathrm{~Hz}$ ), 3.04 (dd, 1H, 7.1, 1.7 Hz), 2.42 (dddd, 1H, 13.1, 9.1, 9.1, 7.2 Hz), 2.25 (dddd, 1H, 13.1, 7.9, 2.0, 2.0 Hz), 0.21 (s, 9H), $0.18(\mathrm{~s}, 9 \mathrm{H})$, and $0.17(\mathrm{~s}, 9 \mathrm{H})$.

${ }^{13} \mathrm{C}-\mathrm{NMR}\left(125 \mathrm{MHz}, \mathrm{CDCl}_{3}\right): \delta 171.9,170.0,95.8,78.9,77.4,53.6,52.0,40.9,28.5,1.9,0.5$, and 0.4 .

IR: 2958, 2902, and $1734 \mathrm{~cm}^{-1}$.

MS: ESI-MS calcd for $\mathrm{C}_{18} \mathrm{H}_{37} \mathrm{NO}_{6} \mathrm{Si}_{3} \mathrm{Na}\left(\mathrm{M} \bullet \mathrm{Na}^{+}\right)$470.1821, found 470.1826. 


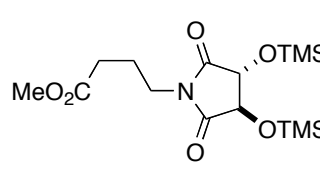

$20 a$

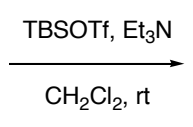

$\mathrm{CH}_{2} \mathrm{Cl}_{2}$, rt

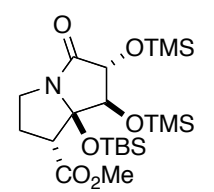

$21 b$

Methyl $(1 R, 6 R, 7 R, 7 \mathrm{a} R)-7 \mathrm{a}-[[(1,1-D i m e t h y l e t h y l) d i m e t h y l s i l y l] 0 x y]$ hexahydro-5-0xo-6,7bis[(trimethylsilyl)oxy]-1H-pyrrolizine-1-carboxylate (21b).

L-Imide ester 20a (300 mg, $0.8 \mathrm{mmol})$ was dissolved in $\mathrm{CH}_{2} \mathrm{Cl}_{2}(3 \mathrm{~mL})$. $\mathrm{Et}_{3} \mathrm{~N}(390 \mu \mathrm{L}, 3.6$ mmol) and TBSOTf $(550 \mu \mathrm{L}, 3.2 \mathrm{mmol})$ were added sequentially to the reaction mixture at $0{ }^{\circ} \mathrm{C}$. The mixture was warmed to rt and stirred for $2 \mathrm{~h}$. Additional $\mathrm{Et}_{3} \mathrm{~N}(220 \mu \mathrm{L}, 1 . .6 \mathrm{mmol})$ and TBSOTf $(280 \mu \mathrm{L}, 1.2 \mathrm{mmol})$ were added. The resulting mixture was stirred at $\mathrm{rt}$ for $20 \mathrm{~h}$ and diluted with $\mathrm{CH}_{2} \mathrm{Cl}_{2}(20 \mathrm{~mL})$. This solution was washed sequentially with saturated aqueous $\mathrm{NaHCO}_{3}$ and brine and dried over $\mathrm{Na}_{2} \mathrm{SO}_{4}$. GC-MS analysis of this crude mixture indicated a 24:1 ratio of two diastereomers. MPLC on silica gel with hexanes/EtOAc 6:1 as eluent gave 21b (297 mg, $0.61 \mathrm{mmol}, 76 \%)$ as a colorless oil having an $[\alpha]_{\mathrm{D}}=+58.5^{\circ}\left(\mathrm{c} 0.68, \mathrm{CHCl}_{3}\right)$. Lit. $^{5}$ : $[\alpha]_{\mathrm{D}}=+51.6^{\circ}\left(\mathrm{c} 1.0, \mathrm{CHCl}_{3}\right)$.

For 21b:

TLC: $\mathrm{R}_{\mathrm{f}}$ (hexanes/EtOAc, 6:1) $=0.30$.

${ }^{1} \mathrm{H}-\mathrm{NMR}\left(500 \mathrm{MHz}, \mathrm{CDCl}_{3}\right): \delta 4.45$ (ddd, $\left.1 \mathrm{H}, J=8.0,1.0,1.0 \mathrm{~Hz}\right), 3.91(\mathrm{~d}, 1 \mathrm{H}, J=8.0 \mathrm{~Hz}$ ), 3.77 (ddd, $1 \mathrm{H}, J=11.0,8.5,8.5 \mathrm{~Hz}$ ), 3.69 (s, $3 \mathrm{H}$ ), 3.25 (dddd, $1 \mathrm{H}, J=10.5,9.0,3.0,1.5 \mathrm{~Hz}$ ), $3.03(\mathrm{dd}, 1 \mathrm{H}, J=7.0,2.5 \mathrm{~Hz}$ ), 2.41 (dddd, $1 \mathrm{H}, J=13.0,9.0,9.0,7.5 \mathrm{~Hz}), 2.24$ (dddd, $1 \mathrm{H}, J=$ 13.5, 8.0, 3.0, 3.0 Hz), $0.88(\mathrm{~s}, 9 \mathrm{H}), 0.21(\mathrm{~s}, 9 \mathrm{H}), 0.17(\mathrm{~s}, 9 \mathrm{H}), 0.16(\mathrm{~s}, 3 \mathrm{H})$, and $0.12(\mathrm{~s}, 3 \mathrm{H})$.

GC-MS (Start temp $=50{ }^{\circ} \mathrm{C}$, run time $=21 \mathrm{~min}$, temp. $\mathrm{ramp}=11.4{ }^{\circ} \mathrm{C} / \mathrm{min}$ ): $\mathrm{t}_{\mathrm{R}}=12.9 \mathrm{~min} ; \mathrm{m} / \mathrm{z}$ : $343\left(\mathrm{M}^{+}\right), 328\left(\mathrm{M}^{+}-\mathrm{CH}_{3}\right)$. 


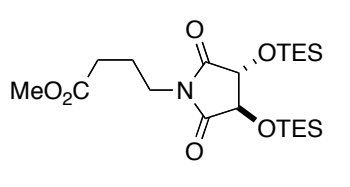

$20 b$

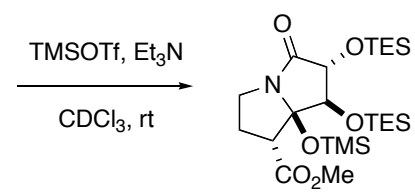

$21 c$

\section{Methyl (1R,6R,7R,7aR)-7a-[(Trimethylsilyl)oxy]hexahydro-5-oxo-6,7-bis[(triethylsilyl)oxy]-} 1H-pyrrolizine-1-carboxylate (21c)

To a solution of L-imide ester $\mathbf{2 0 b}(0.060 \mathrm{~g}, 0.131 \mathrm{mmol})$ in $\mathrm{CDCl}_{3}(0.6 \mathrm{~mL}), \mathrm{Et}_{3} \mathrm{~N}(54 \mu \mathrm{L}, 0.388$ $\mathrm{mmol})$ and TMSOTf ( $63 \mu \mathrm{L}, 0.318 \mathrm{mmol})$ were added sequentially. This mixture was kept at ambient temperature and monitored by ${ }^{1} \mathrm{H}$ NMR spectroscopy. After $48 \mathrm{~h}$ the reaction mixture (dr 23:1 by GC-MS) was diluted with $\mathrm{CH}_{2} \mathrm{Cl}_{2}\left(15 \mathrm{~mL}\right.$ ), washed sequentially with aq $\mathrm{NaHCO}_{3}$ $(2 \times 15 \mathrm{~mL})$ and brine $(2 \times 10 \mathrm{~mL})$, dried over $\mathrm{Na}_{2} \mathrm{SO}_{4}$, concentrated in vacuo, and purified by silica gel chromatography with hexanes/EtOAc $6: 1$ as eluent to give $21 \mathrm{c}(0.040 \mathrm{~g}, 58 \%)$ as a colorless oil.

$[\alpha]_{\mathrm{D}}=+61.0^{\circ}\left(\mathrm{c} 0.10, \mathrm{CHCl}_{3}\right)$.

TLC: $\mathrm{R}_{\mathrm{f}}($ hexanes/EtOAc, 6:1) $=0.35$.

${ }^{1} \mathrm{H}-\mathrm{NMR}\left(500 \mathrm{MHz}, \mathrm{CDCl}_{3}\right)$ : $\delta 4.51$ (ddd, $\left.1 \mathrm{H}, J=7.0,1,1 \mathrm{~Hz}\right), 3.91$ (d, $\left.1 \mathrm{H}, J=7.0 \mathrm{~Hz}\right), 3.79$ (ddd, $1 \mathrm{H}, J=10.4,10.4,7.9 \mathrm{~Hz}$ ), 3.66 (s, $3 \mathrm{H}), 3.22$ (dddd, $1 \mathrm{H}, J=11.1,9.3,1.4,1.4 \mathrm{~Hz}$ ), 3.02 (d, $1 \mathrm{H}, J=7.0 \mathrm{~Hz}$ ), 2.44 (dddd, $1 \mathrm{H}, J=13.3,9.7,9.7,7.2 \mathrm{~Hz}$ ), 2.22 (dddd, $1 \mathrm{H}, J=13.2,7.6,1.3$, $1.3 \mathrm{~Hz}$ ), 0.99 (t, $18 \mathrm{H}, J=8.0 \mathrm{~Hz}$ ), 0.88 (ddd, $3 \mathrm{H}, J=15.2,7.6,7.6 \mathrm{~Hz}), 0.83$ (ddd, $3 \mathrm{H}, J=16.2$, $8.0,8.0 \mathrm{~Hz}), 0.81(\mathrm{q}, 6 \mathrm{H}, J=7.9 \mathrm{~Hz})$, and $0.14(\mathrm{~s}, 9 \mathrm{H})$.

${ }^{13} \mathrm{C}-\mathrm{NMR}\left(125 \mathrm{MHz}, \mathrm{CDCl}_{3}\right): \delta$ 172.3, 171.1, 95.7, 79.8, 77.1, 54.4, 52.0, 41.6, 28.3, 6.93, 6.85, $5.2,4.9$, and 1.5 .

IR: 2956, 2913, 2879, 1737, 1249, 1149, 843, and $744 \mathrm{~cm}^{-1}$.

MS: ESI-MS calcd for $\mathrm{C}_{24} \mathrm{H}_{49} \mathrm{NNaO}_{6} \mathrm{Si}_{3}\left(\mathrm{M} \cdot \mathrm{Na}^{+}\right)$554.2760, found 554.2744. 


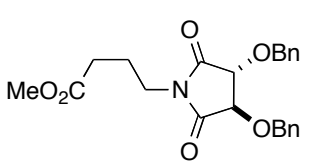

$20 c$

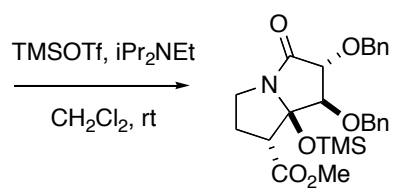

21d

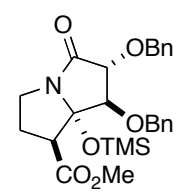

22d

Methyl (1R,6R,7R,7aR)-7a-[(Trimethylsilyl)oxy]hexahydro-5-oxo-6,7-bis(benzyloxy)-1Hpyrrolizine-1-carboxylate (21d) and Methyl (1S,6R,7R,7aS)-7a[(Trimethylsilyl)oxy]hexahydro-5-oxo-6,7-bis[(benzyl)oxy]-1H-pyrrolizine-1-carboxylate (22d).

L-Imide 20c (74 mg, $0.18 \mathrm{mmol})$ was dissolved in $\mathrm{CDCl}_{3}(0.6 \mathrm{~mL})$. $i \operatorname{Pr}_{2} \mathrm{NEt}(103 \mu \mathrm{L}, 0.59$ $\mathrm{mmol}$ ) and TMSOTf ( $98 \mu \mathrm{L}, 0.54 \mathrm{mmol})$ were added sequentially to the reaction mixture at $\mathrm{rt}$ and stirred for $4 \mathrm{~h}$. Additional $i \operatorname{Pr}_{2} \mathrm{NEt}(67 \mu \mathrm{L}, 0.40 \mathrm{mmol})$ and TMSOTf $(66 \mu \mathrm{L}, 0.36 \mathrm{mmol})$ were added. The resulting mixture was stirred at rt for $40 \mathrm{~h}$, diluted with $\mathrm{CH}_{2} \mathrm{Cl}_{2}(20 \mathrm{~mL})$, and washed sequentially with a saturated aqueous solution of $\mathrm{NaHCO}_{3}$ and brine. Organic layer was dried over $\mathrm{Na}_{2} \mathrm{SO}_{4}$. The solution was concentrated and purified by silica gel chromatography with hexanes/EtOAc 4:1 as eluent, yielding a mixture (76 $\mathrm{mg}, 87 \%$ ) of $\mathbf{2 1 d}$ and $\mathbf{2 2 d}$ in 11:1 ratio as a colorless oil.

For the major diastereomer 21d:

$[\alpha]_{\mathrm{D}}=+64.7^{\circ}\left(\mathrm{c} 0.36, \mathrm{CDCl}_{3}\right)$.

TLC: $\mathrm{R}_{\mathrm{f}}($ hexanes/EtOAc, $3: 1)=0.35$.

${ }^{1} \mathrm{H}-\mathrm{NMR}\left(500 \mathrm{MHz}, \mathrm{CDCl}_{3}\right)$ : $\delta 7.39-7.27(\mathrm{~m}, 10 \mathrm{H}), 4.99(\mathrm{~d}, 1 \mathrm{H}, J=12.0 \mathrm{~Hz}), 4.76(\mathrm{~d}, 1 \mathrm{H}, J=$ $12.0 \mathrm{~Hz}), 4.70(\mathrm{~d}, 1 \mathrm{H}, J=11.5 \mathrm{~Hz}), 4.49(\mathrm{dt}, 1 \mathrm{H}, J=8.0,1.2 \mathrm{~Hz}), 4.46(\mathrm{~d}, 1 \mathrm{H}, J=11.0 \mathrm{~Hz}), 3.86$ (d, $1 \mathrm{H}, J=7.5 \mathrm{~Hz}$ ), 3.82 (ddd, $1 \mathrm{H}, J=10.6,8.6,8.6 \mathrm{~Hz}$ ), 3.57 (s, $3 \mathrm{H}$ ), 3.26 (dddd, $1 \mathrm{H}, J=11.2$, 9.3, 1.7, $1.7 \mathrm{~Hz}$ ), $3.08(\mathrm{dd}, 1 \mathrm{H}, J=7.1,1.5 \mathrm{~Hz}), 2.45$ (dddd, $1 \mathrm{H}, J=13.1,9.2,9.2,7.3 \mathrm{~Hz}), 2.28$ (dddd, $1 \mathrm{H}, J=13.2,8.0,1.7,1.7 \mathrm{~Hz}$ ), and 0.08 (s, 9H).

${ }^{13} \mathrm{C}-\mathrm{NMR}\left(75 \mathrm{MHz}, \mathrm{CDCl}_{3}\right)$ : $\delta 172.1,170.3,137.9,137.7,128.51,128.49,128.4,128.03$, $127.94,127.91,95.2,82.4,81.0,72.7,72.3,55.1,52.3,41.5,28.6$, and 1.2.

IR: $3063,3031,2954,2901,1731,1721,1355,1115$, and $842 \mathrm{~cm}^{-1}$.

MS: ESI-MS calcd for $\mathrm{C}_{26} \mathrm{H}_{34} \mathrm{NO}_{6} \mathrm{Si}\left(\mathrm{M} \bullet \mathrm{H}^{+}\right)$484.2150, found 484.2150 .

Resonances from the minor isomer consistent with structure 22d (cf. ${ }^{1} \mathrm{H}$ NMR data from 22a): $\delta$ $4.18(\mathrm{~d}, 1 \mathrm{H}, J=4.0 \mathrm{~Hz}) 4.02(\mathrm{~d}, 1 \mathrm{H}, J=4.0 \mathrm{~Hz}), 3.30(\mathrm{~m}, 1 \mathrm{H}), 3.00$ (d, $1 \mathrm{H}, J=8.4 \mathrm{~Hz}), 22.55$ (m, $1 \mathrm{H})$, and $2.10(\mathrm{br} \mathrm{dd}, 1 \mathrm{H}, \Sigma J=21 \mathrm{~Hz})$. 


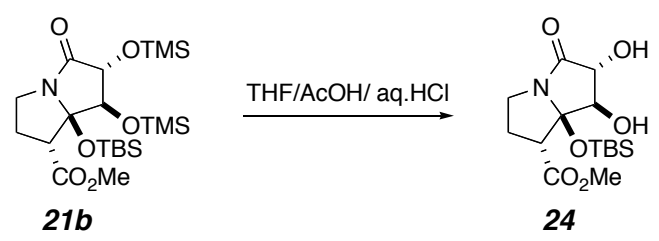

Methyl (1R,6R,7R,7aR)-7a-\{[(1,1-Dimethylethyl)dimethylsilyl $] 0 x y\}$ hexahydro-5-oxo-6,7dihydroxy-1H-pyrrolizine-1-carboxylate (24).

A solution of bis-trimethylsilyl ether $\mathbf{2 1 b}^{5}(220 \mathrm{mg}, 0.45 \mathrm{mmol})$ in THF $(6 \mathrm{~mL})$ was treated with $\mathrm{AcOH}(0.2 \mathrm{~mL})$ and aqueous $\mathrm{HCl}(1.0 \mathrm{M}, 0.2 \mathrm{~mL})$ at $0{ }^{\circ} \mathrm{C}$. The resulting solution was stirred for $20 \mathrm{~min}$ at $\mathrm{rt}$, diluted with EtOAc $(20 \mathrm{~mL})$, washed sequentially with water $(2 \times 2 \mathrm{~mL})$ and aqueous $\mathrm{NaHCO}_{3}(1 \mathrm{~mL})$, dried over $\mathrm{Na}_{2} \mathrm{SO}_{4}$, and concentrated in vacuo. The resulting residue was purified by silica gel chromatography with EtOAc as eluent, yielding pure 24 (65 mg, 42\%) as white crystals.

$\mathrm{mp}=111-115^{\circ} \mathrm{C}$.

$[\alpha]_{\mathrm{D}}=+17.0^{\circ}(\mathrm{c} 0.27, \mathrm{MeOH})$.

TLC: $\mathrm{R}_{\mathrm{f}}($ hexanes/EtOAc, $1: 1)=0.20$.

${ }^{1} \mathrm{H}-\mathrm{NMR}\left(500 \mathrm{MHz}, \mathrm{CDCl}_{3}\right): \delta 4.40(\mathrm{ddd}, 1 \mathrm{H}, J=8.5,3.3,1.0 \mathrm{~Hz}), 3.98(\mathrm{ddd}, 1 \mathrm{H}, J=11.9$, 7.8, $3.7 \mathrm{~Hz}$ ), 3.89 (dd, 1H, $J=8.7,8.5 \mathrm{~Hz}$ ), 3.76 (s, 3H), 3.19 (dddd, 1H, $J=11.4,8.8,6.9,1.3$ $\mathrm{Hz}), 3.14(\mathrm{dd}, 1 \mathrm{H}, J=8.9,7.4 \mathrm{~Hz}), 2.85(\mathrm{~d}, 1 \mathrm{H}, J=8.9 \mathrm{~Hz}), 2.61(\mathrm{~d}, 1 \mathrm{H}, J=3.3 \mathrm{~Hz}), 2.28$ (dddd, $1 \mathrm{H}, J=13.3,7.5,6.9,3.7 \mathrm{~Hz}$ ), 2.17 (dddd, $1 \mathrm{H}, J=13.1,8.7,8.7,7.7 \mathrm{~Hz}), 0.94$ (s, 9H), $0.22(\mathrm{~s}, 3 \mathrm{H})$, and $0.15(\mathrm{~s}, 3 \mathrm{H})$.

${ }^{13} \mathrm{C}-\mathrm{NMR}\left(125 \mathrm{MHz}, \mathrm{CDCl}_{3}\right): \delta 173.5,170.7,95.4,78.8,76.2,54.1,52.8,43.0,29.3,25.8,18.3$, -3.15 and -3.05 .

IR: 3425, 3292, 2953, 2930, 2859, 1732, 1713, 1354, 1291, 1223, and $1120 \mathrm{~cm}^{-1}$.

MS: ESI-MS calcd for $\mathrm{C}_{15} \mathrm{H}_{27} \mathrm{NNaO}_{6} \mathrm{Si}\left(\mathrm{M} \cdot \mathrm{Na}^{+}\right) 368.1500$, found 368.1499.

A sample for single crystal x-ray analysis was obtained by dissolving $20 \mathrm{mg}$ of $24 \mathrm{in} \sim 1.0 \mathrm{~mL}$ of ethyl acetate in a small test tube. The tube was immersed in a larger screw-capped jar containing hexanes to a depth of $\sim 1 \mathrm{~cm}$. The jar was capped and stored at room temperature. After several days, suitable crystals had grown inside the tube. 


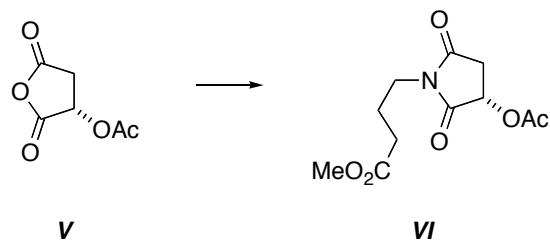

\section{Methyl (3S)-3-Acetyloxy-2,5-dioxo-1-pyrrolidinebutanoate (VI).}

Anhydride $\mathbf{V}^{7}$ (1.19 g, $7.5 \mathrm{mmol}$ ) was dissolved in $\mathrm{CH}_{3} \mathrm{CN}$ and the hydrochloride salt of methyl 4-aminobutyrate $(1.21 \mathrm{~g}, 7.9 \mathrm{mmol})$ was added followed by $\mathrm{Et}_{3} \mathrm{~N}(1.1 \mathrm{~mL}, 7.9 \mathrm{mmol})$. The reaction mixture was stirred for $2 \mathrm{~h}$, and the solvent was removed in vacuo. Acetyl chloride (15 $\mathrm{mL}$ ) was added and the reaction mixture was stirred for $2 \mathrm{~h}$. Excess acetyl chloride was removed by distillation (at one atmosphere), and the residue was chromatographed on silica gel with hexanes/EtOAc 2:1 as eluent. Imide VI (1.30 g, $5.1 \mathrm{mmol}, 67 \%)$ was obtained as pale yellow oil. $[\alpha]_{\mathrm{D}}=-12.5^{\circ}\left(\mathrm{c} 3.12, \mathrm{CHCl}_{3}\right)$.

TLC: $\mathrm{R}_{\mathrm{f}}$ (hexanes/EtOAc, $\left.1: 1\right)=0.29$.

${ }^{1} \mathrm{H}-\mathrm{NMR}\left(500 \mathrm{MHz}, \mathrm{CDCl}_{3}\right): \delta 5.40(\mathrm{dd}, 1 \mathrm{H}, J=8.7,4.8 \mathrm{~Hz}), 3.68(\mathrm{~s}, 3 \mathrm{H}), 3.63(\mathrm{ddd}, 1 \mathrm{H}, J=$ $13.5,6.7,6.7 \mathrm{~Hz}$ ), 3.62 (ddd, $1 \mathrm{H}, J=13.6,6.7,6.7 \mathrm{~Hz}), 3.15$ (dd, 1H, $J=18.3,8.8 \mathrm{~Hz}$ ), 2.68 (dd, $1 \mathrm{H}, J=18.3,4.5 \mathrm{~Hz}$ ), 2.37 (t, 2H, $J=7.3 \mathrm{~Hz}$ ), 2.17 (s, 3H), and 1.95 (pent, $2 \mathrm{H}, J=7 \mathrm{~Hz}$ ).

${ }^{13} \mathrm{C}-\mathrm{NMR}\left(75 \mathrm{MHz}, \mathrm{CDCl}_{3}\right): \delta 173.7,173.4,173.1,170.0,67.7,51.8,38.4,35.7,31.3,22.8$, and 20.6 .

IR: 2953, $1714 \mathrm{~cm}^{-1}$.

MS: ESI-MS calcd for $\mathrm{C}_{11} \mathrm{H}_{15} \mathrm{NO}_{6} \mathrm{Na}\left(\mathrm{M} \bullet \mathrm{Na}^{+}\right)$280.0792, found 280.0790. 


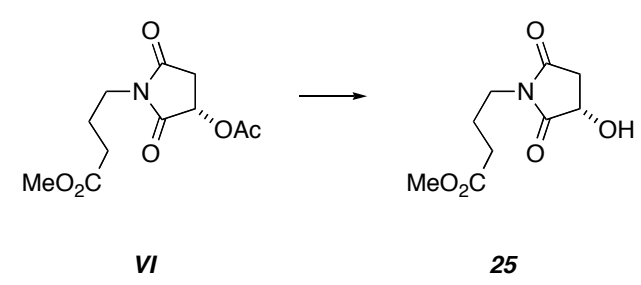

\section{Methyl (3S)-3-Hydroxy-2,5-dioxo-1-pyrrolidinebutanoate (25).}

Acetate VI $(287 \mathrm{mg}, 1.12 \mathrm{mmol})$ was dissolved in methanol $(20 \mathrm{~mL})$ and acetyl chloride $(10 \mu \mathrm{L})$ was added while stirring. The resulting reaction mixture was heated under reflux for $48 \mathrm{~h}$, cooled, and concentrated under reduced pressure to produce 25 as a clear oil (208 $\mathrm{mg}, 0.97$ mmol, 86\%) that was of excellent purity ( ${ }^{1} \mathrm{H}$ NMR and TLC analyses).

$[\alpha]_{\mathrm{D}}=-56.0^{\circ}\left(\mathrm{c} 3.16, \mathrm{CHCl}_{3}\right)$.

TLC: $\mathrm{R}_{\mathrm{f}}($ EtOAc $)=0.54$.

${ }^{1} \mathrm{H}-\mathrm{NMR}\left(500 \mathrm{MHz}, \mathrm{CDCl}_{3}\right): \delta 4.63(\mathrm{~m}, 1 \mathrm{H}), 3.68(\mathrm{~s}, 3 \mathrm{H}), 3.60$ (ddd, 1H, J=13.5, 6.8, $6.8 \mathrm{~Hz}$ ), 3.59 (ddd, $1 \mathrm{H}, J=13.5,6.7,6.7 \mathrm{~Hz}), 3.06(\mathrm{dd}, 1 \mathrm{H}, J=18.2,8.4 \mathrm{~Hz}), 2.97$ (br s, 1H), 2.68 (dd, $1 \mathrm{H}, J=18.2,4.7 \mathrm{~Hz}$ ), 2.36 (t, 2H, $J=7.2 \mathrm{~Hz}$ ), and 1.94 (pent, $2 \mathrm{H}, J=7.1 \mathrm{~Hz}$ ).

${ }^{13} \mathrm{C}-\mathrm{NMR}\left(125 \mathrm{MHz}, \mathrm{CDCl}_{3}\right): \delta 178.8,174.7,173.3,66.9,51.9,38.1,37.4,31.3$, and 22.8.

IR: 3461 (br), 2953, 1725 (sh), and $1703 \mathrm{~cm}^{-1}$.

MS: ESI-MS calcd for $\mathrm{C}_{9} \mathrm{H}_{13} \mathrm{NO}_{5} \mathrm{Na}\left(\mathrm{M} \bullet \mathrm{Na}^{+}\right)$238.0686, found 238.0687. 


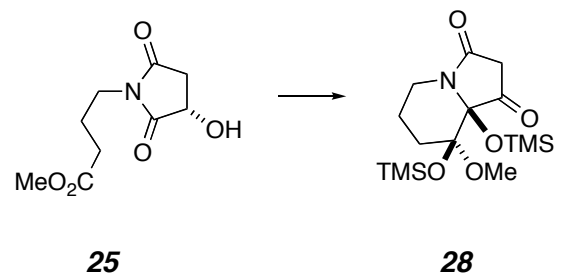

(4R,5R)-rel-5-Methoxy-4,5-bis[(trimethylsilyl)oxy]-1,3(2H,5H)-indolizinedione (28).

Malimide 25 (39 mg, $0.18 \mathrm{mmol})$ was dissolved in $\mathrm{CDCl}_{3}(0.5 \mathrm{~mL})$ and the solution was transferred to an NMR tube. $\mathrm{Et}_{3} \mathrm{~N}(125 \mu \mathrm{L}, 0.90 \mathrm{mmol})$ was added and the tube was shaken to ensure proper mixing. TMSOTf $(129 \mu \mathrm{L}, 0.72 \mathrm{mmol})$ was added and the tube was capped and shaken well. After the disappearance of the in situ and immediately formed $O$-TMS ether of 25 was observed (ca. $1 \mathrm{~h}$ ) by ${ }^{1} \mathrm{H}$ NMR analysis (by disappearance of CHOTMS), the solution was partitioned between $5 \%$ aqueous $\mathrm{NaHCO}_{3}(30 \mathrm{~mL})$ and $\mathrm{CH}_{2} \mathrm{Cl}_{2}(40 \mathrm{~mL})$. The aqueous layer was extracted with more $\mathrm{CH}_{2} \mathrm{Cl}_{2}(2 \times 20 \mathrm{~mL})$ and dried over $\mathrm{Na}_{2} \mathrm{SO}_{4}$. This extract was concentrated and GC-MS analysis of the residue showed only one peak with $m / z$ corresponding to the molecular ion and the fragmentation pattern consistent with $\mathbf{2 8}$. The residue was filtered through a plug of silica gel with hexanes/EtOAc/ $\mathrm{Et}_{3} \mathrm{~N} \mathrm{40:10:1} \mathrm{as} \mathrm{eluent} \mathrm{to} \mathrm{give} 28$ (55 $\mathrm{mg}, 0.18 \mathrm{mmol}$, $100 \%$, d.r. $14: 1^{8}$ ) that was ca. $90 \%$ pure by ${ }^{1} \mathrm{H}$ NMR analysis. Attempts to further purify 28 on a preparative MPLC column with the same eluent resulted in $24 \mathrm{mg}$ (44\% recovery) of 28 having essentially the same level of purity. Such behavior suggests decomposition on silica gel.

$[\alpha]_{\mathrm{D}}=-2.1^{\circ}\left(\mathrm{c} 1.17, \mathrm{CHCl}_{3}\right)$. If it arises entirely by the mechanism offered in Scheme 7 of the manuscript, then the sample of $\mathbf{2 8}$ should be racemic. We would suggest that the observed small rotation arises from residual amounts of chiral, non-racemic impurities. The lability of this silyloxy ketal precluded more extensive purification.

TLC: $\mathrm{R}_{\mathrm{f}}$ (hexanes/EtOAc, $\left.1: 1\right)=0.66$.

${ }^{1} \mathrm{H}-\mathrm{NMR}\left(500 \mathrm{MHz}, \mathrm{CDCl}_{3}\right): \delta 4.21(\mathrm{ddd}, 1 \mathrm{H}, J=13.2,4.4,1.5 \mathrm{~Hz}), 3.11(\mathrm{~s}, 3 \mathrm{H}), 3.06(\mathrm{~d}, 1 \mathrm{H}, J$ $=21.4 \mathrm{~Hz}), 2.82(\mathrm{dd}, 1 \mathrm{H}, J=22.0,1.5 \mathrm{~Hz}), 2.77$ (dddd, $1 \mathrm{H}, J=13.2,13.2,2.9,1.5 \mathrm{~Hz}), 1.94-$

1.91 [nfom, $2 \mathrm{H}\left(J_{\mathrm{S}}\right.$ from spectrum in $1: 1 \mathrm{C}_{6} \mathrm{D}_{6}: \mathrm{CDCl}_{3}$ are: ddd, $1 \mathrm{H}, 13.7,13.7,3.9 \mathrm{~Hz}$ and dddd, $1 \mathrm{H}, 13.9,4.0,3.0,1.1 \mathrm{~Hz})$ ], 1.68 [ddddd, $1 \mathrm{H}, J=13.2,13.213 .2,5.1,3.9 \mathrm{~Hz}$ (Js from spectrum in $\left.1: 1 \mathrm{C}_{6} \mathrm{D}_{6}: \mathrm{CDCl}_{3}\right)$ ], 1.56 (ddddd, $\left.1 \mathrm{H}, J=13.3,3.6,3.6,3.6,1.5 \mathrm{~Hz}\right), 0.21(\mathrm{~s}, 9 \mathrm{H})$, and $0.13(\mathrm{~s}$, 9H).

${ }^{13} \mathrm{C}-\mathrm{NMR}\left(125 \mathrm{MHz}, \mathrm{CDCl}_{3}\right): \delta 204.2,168.2,105.0,100.1,48.2,41.8,35.4,28.1,22.3,2.3$, and 1.4.

IR: $2957,1782,1711 \mathrm{~cm}^{-1}$.

MS: ESI-MS calcd for $\mathrm{C}_{15} \mathrm{H}_{29} \mathrm{NO}_{5} \mathrm{Si}_{2} \mathrm{Na}\left(\mathrm{M} \bullet \mathrm{Na}^{+}\right)$382.1476, found 382.1495. 


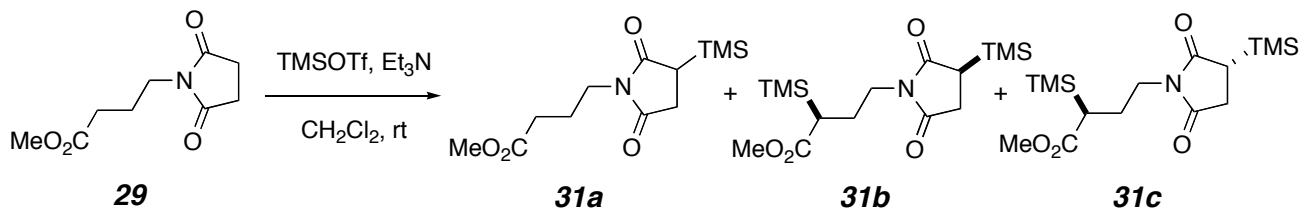

Methyl 2,5-Dioxo-3-trimethylsilyl-1-pyrrolidinebutanoate (31a), Methyl (3R, $\alpha R)$-rel-2,5Dioxo-3-trimethylsilyl-1-pyrrolidine( $\alpha$-trimethylsilyl)butanoate (31b) and Methyl (3S, $\alpha R)$ rel-2,5-Dioxo-3-trimethylsilyl-1-pyrrolidine( $\alpha$-trimethylsilyl)butanoate (31c).

Succinimide ester 29 (40 mg, $0.24 \mathrm{mmol})$ was dissolved in $\mathrm{CDCl}_{3}(0.5 \mathrm{~mL})$, and $\mathrm{Et}_{3} \mathrm{~N}(100 \mu \mathrm{L}$, $0.7 \mathrm{mmol})$ and TMSOTf $(126 \mu \mathrm{L}, 0.69 \mathrm{mmol})$ were added at $\mathrm{rt}$. The reaction mixture was stirred for $24 \mathrm{~h}$, and additional $\mathrm{Et}_{3} \mathrm{~N}(55 \mu \mathrm{L}, 0.22 \mathrm{mmol})$ and TMSOTf $(72 \mu \mathrm{L}, 0.20 \mathrm{mmol})$ were added. The mixture was stirred at $\mathrm{rt}$ for $48 \mathrm{~h}$, diluted with $\mathrm{CH}_{2} \mathrm{Cl}_{2}(10 \mathrm{~mL})$, and washed sequentially with a saturated aqueous solution of $\mathrm{NaHCO}_{3}$ and brine. The organic layer was dried over $\mathrm{Na}_{2} \mathrm{SO}_{4}$. The solution was concentrated and purified by silica gel chromatography with hexanes/EtOAc 3:1 as eluent, yielding, in order of elution, 31b (28 mg, $0.088 \mathrm{mmol}, 30 \%), 31 \mathrm{c}$ (26 mg, $0.076 \mathrm{mmol}, 30 \%$ ), and 31a (9 mg, $0.033 \mathrm{mmol}, 13 \%$ ), as colorless oils. Compounds 31b and 31c are separable by silica gel chromatography, however, we could not assign their relative configurations.

For 31b:

TLC: $\mathrm{R}_{\mathrm{f}}($ hexanes/EtOAc, $3: 1)=0.25$.

${ }^{1} \mathrm{H}-\mathrm{NMR}\left(500 \mathrm{MHz}, \mathrm{CDCl}_{3}\right): \delta 3.67$ (s, 3H), 3.51 (app t, 2H, $\left.J=7 \mathrm{~Hz}\right), 2.85(\mathrm{dd}, 1 \mathrm{H}, J=18.5$, $9.8 \mathrm{~Hz}), 2.50(\mathrm{dd}, 1 \mathrm{H}, J=18.8,3.6 \mathrm{~Hz}), 2.35(\mathrm{dd}, 1 \mathrm{H}, J=9.6,3.4 \mathrm{~Hz}), 2.06(\mathrm{dddd}, 1 \mathrm{H}, J=13.6$, $12.2,6.2,6.2 \mathrm{~Hz}), 1.96(\mathrm{dd}, 1 \mathrm{H}, J=12.2,2.7 \mathrm{~Hz}), 1.68$ (dddd, $1 \mathrm{H}, J=13.4,7.4,7.4,2.5 \mathrm{~Hz}$ ), $0.15(\mathrm{~s}, 9 \mathrm{H})$, and $0.06(\mathrm{~s}, 9 \mathrm{H})$.

${ }^{13} \mathrm{C} \mathrm{NMR}\left(75 \mathrm{MHz}, \mathrm{CDCl}_{3}\right): \delta 179.6,177.6,175.0,51.3,38.8,34.9,31.8,31.1,25.0,-2.5$, and 3.0 .

IR: 2954, 2901, 1763, 1692, 1398, 1252, and $1193 \mathrm{~cm}^{-1}$.

MS: ESI-MS calcd for $\mathrm{C}_{15} \mathrm{H}_{29} \mathrm{NNaO}_{4} \mathrm{Si}_{2}\left(\mathrm{M} \cdot \mathrm{Na}^{+}\right)$366.1527, found 366.1527.

GC-MS (Start temp $=50{ }^{\circ} \mathrm{C}$, run time $=21 \mathrm{~min}$, temp. ramp $=11.4{ }^{\circ} \mathrm{C} / \mathrm{min}$ ): $\mathrm{t}_{\mathrm{R}}=11.0 \mathrm{~min} ; \mathrm{m} / \mathrm{z}$ : $343\left(\mathrm{M}^{+}\right), 328\left(\mathrm{M}^{+}-\mathrm{CH}_{3}\right)$.

For 31c:

TLC: $\mathrm{R}_{\mathrm{f}}($ hexanes/EtOAc, $3: 1)=0.22$.

${ }^{1} \mathrm{H}-\mathrm{NMR}\left(500 \mathrm{MHz}, \mathrm{CDCl}_{3}\right.$ ): $\delta 3.69$ (s, 3H), 3.55 (ddd, 1H, $J=13.0,7.3,4.7 \mathrm{~Hz}$ ), 3.48 (ddd, $1 \mathrm{H}, J=13.3,7.3,7.3 \mathrm{~Hz}), 2.84(\mathrm{dd}, 1 \mathrm{H}, J=18.5,9.6 \mathrm{~Hz}), 2.52(\mathrm{dd}, 1 \mathrm{H}, J=18.6,3.2 \mathrm{~Hz}), 2.36$ $(\mathrm{dd}, 1 \mathrm{H}, J=9.7,3.3 \mathrm{~Hz}), 2.05(\mathrm{dddd}, 1 \mathrm{H}, J=13.5,12.0,7.3,4.9 \mathrm{~Hz}), 1.96(\mathrm{dd}, 1 \mathrm{H}, J=12.2,2.6$ $\mathrm{Hz}), 1.67$ (dddd, $1 \mathrm{H}, J=13.5,7.3,7.3,2.4 \mathrm{~Hz}), 0.14(\mathrm{~s}, 9 \mathrm{H})$, and $0.06(\mathrm{~s}, 9 \mathrm{H})$.

${ }^{13} \mathrm{C}-\mathrm{NMR}\left(75 \mathrm{MHz}, \mathrm{CDCl}_{3}\right): \delta 179.7,177.7,175.0,52.0,38.8,34.8,31.3,31.0,25.0,-2.5$, and 3.0.

IR: 2957, 1717, 1693, 1399, 1252, and $1194 \mathrm{~cm}^{-1}$.

MS: ESI-MS calcd for $\mathrm{C}_{15} \mathrm{H}_{29} \mathrm{NNaO}_{4} \mathrm{Si}_{2}\left(\mathrm{M} \cdot \mathrm{Na}^{+}\right)$366.1527, found 366.1531. 
GC-MS (Start temp $=50{ }^{\circ} \mathrm{C}$, run time $=21 \mathrm{~min}$, temp. $\left.\operatorname{ramp}=11.4{ }^{\circ} \mathrm{C} / \mathrm{min}\right): \mathrm{t}_{\mathrm{R}}=11.1 \mathrm{~min} ; \mathrm{m} / \mathrm{z}$ : $343\left(\mathrm{M}^{+}\right), 328\left(\mathrm{M}^{+}-\mathrm{CH}_{3}\right)$.

For 31a:

TLC: $\mathrm{R}_{\mathrm{f}}($ hexanes/EtOAc, $3: 1)=0.12$.

${ }^{1} \mathrm{H}-\mathrm{NMR}\left(500 \mathrm{MHz}, \mathrm{CDCl}_{3}\right.$ ): $\delta 3.67$ (s, 3H), 3.56 (ddd, 1H, $\left.J=13.3,7.0,7.0 \mathrm{~Hz}\right), 3.53$ (ddd, 1H, $J=13.3,7.0,7.0 \mathrm{~Hz}), 2.86(\mathrm{dd}, 1 \mathrm{H}, J=18.6,9.7 \mathrm{~Hz}), 2.51(\mathrm{dd}, 1 \mathrm{H}, J=18.6,3.2 \mathrm{~Hz}), 2.35(\mathrm{dd}$, $1 \mathrm{H}, J=9.7,3.2 \mathrm{~Hz}), 2.31(\mathrm{t}, 2 \mathrm{H}, J=7.4 \mathrm{~Hz}), 1.87$ (pent, $2 \mathrm{H}, J=7.2 \mathrm{~Hz}$ ), and $0.12(\mathrm{~s}, 9 \mathrm{H})$.

${ }^{13} \mathrm{C}-\mathrm{NMR}\left(125 \mathrm{MHz}, \mathrm{CDCl}_{3}\right): \delta$ 179.6, 177.6, 173.2, 51.9, 38.0, 31.8, 31.5, 31.1, 23.3, and -3.0. IR: 2957, 1760, 1738, 1690, 1437, 1399, 1252, and $1175 \mathrm{~cm}^{-1}$.

MS: ESI-MS calcd for $\mathrm{C}_{12} \mathrm{H}_{21} \mathrm{NNaO}_{4} \mathrm{Si}\left(\mathrm{M} \cdot \mathrm{Na}^{+}\right)$294.1132, found 294.1127.

GC-MS (Start temp $=50{ }^{\circ} \mathrm{C}$, run time $=21 \mathrm{~min}$, temp. $\left.\operatorname{ramp}=11.4{ }^{\circ} \mathrm{C} / \mathrm{min}\right): \mathrm{t}_{\mathrm{R}}=11.1 \mathrm{~min} ; \mathrm{m} / \mathrm{z}$ : $271\left(\mathrm{M}^{+}\right), 256\left(\mathrm{M}^{+}-\mathrm{CH}_{3}\right)$.

\section{Supporting Information References}

Fleming, I.; Higgins, D. J. Chem. Soc. Perkin, Trans. 1, 1998, 17, 2673-2678.

2 Yoshifuji, S.; Yukimi, A.; Nitta, Y. Chem. Pharm. Bull. 1985, 33, 5042-5047.

3 Dener, J. M.; Hart, D. J.; Ramesh, S. J. Org. Chem. 1988, 53, 6022-6030.

4 Kukla, M. J.; Breslin, H. J.; Gill, A. J. Med. Chem. 1990, 33, 223-228.

5 Lambert, T. H.; Danishefsky, S. J. J. Am. Chem. Soc. 2006, 128, 426-427.

6 Ohwada, J.; Inouye, Y.; Kimura, M.; Kakisawa, H. Bull. Chem. Soc. Jpn. 1990, 63, 287-289.

7 Mhaske, S. B.; Argade, N. P. J. Org. Chem. 2001, 66, 9038-9040.

8 Resonances consistent with a second (minor) diastereomer were observed in the ${ }^{1} \mathrm{H}$ and ${ }^{13} \mathrm{C}$ NMR spectra; however, a second peak was not observed by capillary GC. 